Original Research Paper

\title{
A New Bivariate Distribution with Generalized Quadratic Hazard Rate Marginals
}

\author{
Abdelfattah Mustafa \\ Department of Mathematics, Faculty of Science, Mansoura University, 35516 Mansoura, Egypt
}

Article history

Received: 24-08-2016

Revised: 25-10-2016

Accepted: 02-11-2016

Email: abdelfatah_mustafa@yahoo.com

\begin{abstract}
Recently, Sarhan (2009) introduced a new distribution named generalized quadratic hazard rate distribution. In this study, we define a bivariate Generalized Quadratic Hazard Rate Distribution (BGQHRD). The joint cumulative distribution function and joint survival function are derived in compact forms. Several properties of BGQFRD have been discussed. The conditional probability density function, $r$ th moments and joint and marginal moment generating functions are obtained. Parameters estimators using the maximum likelihood method are obtained. A numerical illustration is used to obtain maximum likelihood estimators (MLEs). Moreover, we study the behavior of the estimators numerically.
\end{abstract}

Keywords: Bivariate Distributions, Generalized Quadratic Hazard Rate Distribution, Maximum Likelihood Estimators, Information Matrix

\section{Introduction}

Recently, Sarhan (2009) introduced a new distribution named generalized quadratic hazard rate distribution. This paper introduces a Bivariate Generalized Quadratic Hazard Rate Distribution (BGQHRD) by using the method of Marshall and Olkin (1986).

The Generalized Quadratic Hazard Rate Distribution (GQHRD), generalizes several distributions such as the quadratic hazard rate, the generalized linear failure rate, the generalized exponential and the generalized Rayleigh distributions.

The GQHRD may have an decreasing (increasing) hazard or a bathtub shaped hazard or an upside-down bathtub shaped hazard function. This property enables this distribution to be used in many applications such as in reliability, life testing, survival analysis.

Sarhan and Balakrishnan (2007) discussed Marshall and Olkin bivariate exponential distribution, Al-Khedhairi and El-Gohary (2008) introduced a new class of bivariate Gompertz distributions, Kundu and Gupta (2009) studied the bivariate generalized exponential distribution, El-Sherpieny et al. (2013) expressed a new bivariate generalized Gompertz distribution and Kundu and Gupta (2013) presented Marshall-Olkin bivariate Weibull distribution.

The random variable $\mathrm{X}$ has the Quadratic Hazard Rate Distribution (QHRD) with parameters $a, b, c$ if its Cumulative Distribution Function (CDF) is:

$$
F(x ; a, b, c)=1-\exp \left\{-\left(a x+\frac{b}{2} x^{2}+\frac{c}{3} x^{3}\right)\right\}
$$

where, $a \geq 0, c \geq 0$ and $b \geq-2 \sqrt{a c}$. This restriction on the parameter space is made to be insure that the hazard function for QHRD is positive, see Bain (1974):

$$
h(x ; a, b, c)=a+b x+c x^{2}, x \geq 0
$$

The exponential distribution $(\operatorname{ED}(a))$ can be obtained from $\mathrm{QHRD}(\mathrm{a}, \mathrm{b}, \mathrm{c})$ when $b=0, c=0$. We can get the Rayleigh distribution $(\mathrm{RD}(\mathrm{b}))$ from $\mathrm{QHRD}$ when $a=0, c$ $=0$, the Weibull with shape parameter equals 3 $(\mathrm{WD}(\mathrm{c}, 3))$ can be concluded from $\operatorname{QHRD}(\mathrm{a}, \mathrm{b}, \mathrm{c})$ when $a$ $=0, b=0$ and linear hazard rate distribution $(\operatorname{LFRD}(\mathrm{a}, \mathrm{b}))$ can be derived from QHRD $(\mathrm{a}, \mathrm{b}, \mathrm{c})$ when $c=0$.

Sarhan (2009) introduced the generalized quadratic hazard rate distribution with parameters $\mathrm{a}, \mathrm{b}, \mathrm{c}$ and $\mathrm{d}$, $(\operatorname{GQHRD}(\mathrm{a}, \mathrm{b}, \mathrm{c}, \mathrm{d}))$. The $\operatorname{GQHRD}(\mathrm{a}, \mathrm{b}, \mathrm{c}, \mathrm{d})$ has the following $\mathrm{CDF}$ :

$$
F(x ; a, b, c)=\left[1-\exp \left\{-\left(a x+\frac{b}{2} x^{2}+\frac{c}{3} x^{3}\right)\right\}\right]^{d}, x \geq 0
$$

where, $a \geq 0, c \geq 0$ and $b \geq-2 \sqrt{a c}$.

The main objective of this article is to introduce and study the bivariate generalized quadratic hazard rate 
distribution. The BGQHRD generalizes the following distributions:

- The bivariate generalized LFRD when $c=0$

- The bivariate generalized ED, when $b=0, c=0, a>0$

- The bivariate generalized $\mathrm{RD}$, when $a=0, c=0, b>0$

This paper can be organized as follows. Some properties of the BGQHRD are introduced in section 2. Section 3 gives the mathematical expectations. Section 4 presents the moment generating functions. The parameter estimations using maximum likelihood is given in section 5. A set of real data is used as an application in section 6 .

\section{A New Bivariate Generalized Quadratic Hazard Rate Distribution}

In this section, we discuss the BGQHRD. We start with the joint cumulative distribution function of the distribution.

Let $X$ be a random variable has $\mathrm{GQHR}$ distribution with parameters $a, b, c$ and $d$, if its PDF and CDF, respectively, is:

$$
\begin{aligned}
& f(x ; a, b, c, \alpha)=\left(a+b x+c x^{2}\right) e^{-\left(a x+\frac{b}{2} x^{2}+\frac{c}{3} x^{3}\right)} \times \\
& {\left[1-e^{-\left(a x+\frac{b}{2} x^{2}+\frac{c}{3} x^{3}\right)}\right]^{\alpha-1}} \\
& F(x ; a, b, c, \alpha)=\left[1-e^{-\left(a x+\frac{b}{2} x^{2}+\frac{c}{3} x^{3}\right)}\right]^{\alpha}
\end{aligned}
$$

where, $\alpha>, a \geq 0, c \geq 0$ and $b \geq-2 \sqrt{a c}$.

\section{The Joint Cumulative Distribution Function}

Suppose that $U_{1} \sim \operatorname{GQHRD}\left(\alpha_{1}, \mathrm{a}, \mathrm{b}, \mathrm{c}\right), U_{2} \sim \operatorname{GQHRD}\left(\alpha_{2}\right.$, a,b,c) and $U_{3} \sim \operatorname{GQHRD}\left(\alpha_{3}, \mathrm{a}, \mathrm{b}, \mathrm{c}\right)$ are independently distributed.

Define $X_{1}=\max \left(U_{1}, U_{3}\right)$ and $X_{2}=\max \left(U_{2}, U_{3}\right)$. The bivariate vector $\left(X_{1}, X_{2}\right)$ has a BGQHRD with parameters $\alpha_{1}, \alpha_{2}, \alpha_{3}, a, b$ and $c$.

\section{Lemma 1}

The joint cumulative distribution function of $\left(X_{1}, X_{2}\right)$ is given as:

$$
\begin{aligned}
& F_{X_{1}, X_{2}}\left(x_{1}, x_{2}\right)=\left[1-e^{-\left(a x_{1}+\frac{b}{2} x_{1}^{2}+\frac{c}{3} x_{1}^{3}\right)}\right]^{\alpha_{1}}\left[1-e^{-\left(a x_{2}+\frac{b}{2} x_{2}^{2}+\frac{c}{3} x_{2}^{3}\right)}\right]^{\alpha_{2}} \\
& \times\left[1-e^{-\left(a z+\frac{b}{2} z^{2}+\frac{c}{3} z^{3}\right)}\right]^{\alpha_{3}}
\end{aligned}
$$

where, $z=\min \left(x_{1}, x_{2}\right)$.

Proof

Since $F\left(x_{1}, x_{2}\right)=P\left[X_{1} \leq x_{1}, X_{2} \leq x_{2}\right]$, we get:

$$
\begin{aligned}
& F\left(x_{1}, x_{2}\right)=P\left[\max \left(U_{1}, U_{3}\right) \leq x_{1}, \max \left(U_{2}, U_{3}\right) \leq x_{2}\right] \\
& =P\left[U_{1} \leq x_{1}, U_{2} \leq x_{2}, U_{3} \leq \min \left(x_{1}, x_{2}\right)\right]
\end{aligned}
$$

As $U_{i}, i=1,2,3$ are mutually independent, we obtain:

$$
\begin{aligned}
& F\left(x_{1}, x_{2}\right)=P\left[U_{1} \leq x_{1}\right] P\left[U_{2} \leq x_{2}\right] P\left[U_{3} \leq \min \left(x_{1}, x_{2}\right)\right] \perp \\
& =F_{\text {GQHR }}\left(x_{1} ; \alpha_{1}, a, b, c\right) F_{G Q H R}\left(x_{2} ; \alpha_{2}, a, b, c\right) F_{G Q H R}\left(z ; \alpha_{3}, a, b, c\right) .
\end{aligned}
$$

Substituting from Equation 5 into Equation 7, we obtain Equation 6. This completes the proof.

\section{The Joint Probability Density Function}

The following theorem gives the joint PDF of the BGQHRD.

\section{Theorem 2}

If $\left(X_{1}, X_{2}\right)$ has BGQHRD, then the joint probability density function of $\left(X_{1}, X_{2}\right)$ is given by:

$$
f_{X_{1}, X_{2}}\left(x_{1}, x_{2}\right)=\left\{\begin{array}{l}
f_{1}\left(x_{1}, x_{2}\right) \text { if } x_{1}<x_{2}, \\
f_{2}\left(x_{1}, x_{2}\right) \text { if } x_{2}<x_{1}, \\
f_{3}(x, x) \text { if } x_{1}=x_{2}=x .
\end{array}\right.
$$

where:

$$
\begin{aligned}
& f_{1}\left(x_{1}, x_{2}\right)=\left(\alpha_{1}+\alpha_{3}\right)\left(a+b x_{1}+c x_{1}^{2}\right) e^{-\left(a x_{1}+\frac{b}{2} x_{1}^{2}+\frac{c}{3} x_{1}^{3}\right)} \\
& {\left[1-e^{-\left(a x_{1}+\frac{b}{2} x_{1}^{2}+\frac{c}{3} x_{1}^{3}\right)}\right]^{\alpha_{1}+\alpha_{3}-1} \alpha_{2}\left(a+b x_{2}+c x_{2}^{2}\right)} \\
& e^{-\left(a x_{2}+\frac{b}{2} x_{2}^{2}+\frac{c}{3} x_{2}^{3}\right)}\left[1-e^{-\left(a x_{2}+\frac{b}{2} x_{2}+\frac{c}{3} x_{2}^{3}\right)}\right]^{\alpha_{2}-1}
\end{aligned}
$$

$$
f_{2}\left(x_{1}, x_{2}\right)=\alpha_{1}\left(a+b x_{1}+c x_{1}^{2}\right) e^{-\left(a x_{1}+\frac{b}{2} x_{1}^{2}+\frac{c}{3} x_{1}\right)}\left[1-e^{-\left(a x_{1}+\frac{b}{2} x_{1}^{2}+\frac{c}{3} x_{1}^{3}\right.}\right]^{\alpha_{1}-1}\left(\alpha_{2}+\alpha_{3}\right)\left(a+b x_{2}+c x_{2}^{2}\right) e^{-\left(a x_{2}+\frac{b}{2} x_{2}^{2}+\frac{c}{3} x_{2}\right)}\left[1-e^{-\left(a x_{2}+\frac{b}{2} x_{2}^{2}+\frac{c}{3} x_{2}^{3}\right)}\right]^{\alpha_{2}+\alpha_{3}-1}
$$




$$
\begin{aligned}
& f_{3}(x, x)=\alpha_{3}\left(a+b x+c x^{2}\right) e^{-\left(a x+\frac{b}{2} x^{2}+\frac{c}{3} x^{3}\right)} \\
& {\left[1-e^{-\left(a x+\frac{b}{2} x^{2}+\frac{c}{3} x^{3}\right)}\right]^{\alpha_{1}+\alpha_{2}+\alpha_{3}-1} \cdot}
\end{aligned}
$$

\section{Proof}

Let first assume that $x_{1}<x_{2}$, then $F\left(x_{1}, x_{2}\right)$ in Equation 6 will be denoted by $F_{1}\left(x_{1}, x_{2}\right)$ and becomes:

$$
F_{1}\left(x_{1}, x_{2}\right)=\left[1-e^{-\left(a x_{1}+\frac{b}{2} x_{1}^{2}+\frac{c}{3} x_{1}^{3}\right)}\right]^{\alpha_{1}+\alpha_{3}}\left[1-e^{-\left(a x_{2}+\frac{b}{2} x_{2}^{2}+\frac{c}{3} x_{2}^{3}\right)}\right]^{\alpha_{2}}
$$

Differentiating $F_{1}\left(x_{1}, x_{2}\right)$ with respect to $\mathrm{x}_{1}$ and $\mathrm{x}_{2}$, we obtain the expression of $f_{1}\left(x_{1}, x_{2}\right)$ as given in Equation 9. By the same way we can obtain $f_{2}\left(x_{1}, x_{2}\right)$ when $x_{2}<x_{1}$. But $f_{3}\left(x_{1}, x_{2}\right)$ cannot be obtained in a similar way. We can use the following identity to derive $f_{3}(x, x)$ as:

$$
\begin{aligned}
& \int_{0}^{\infty} \int_{0}^{x_{2}} f_{1}\left(x_{1}, x_{2}\right) d x_{1} d x_{2}+\int_{0}^{\infty} \int_{0}^{x_{1}} f_{2}\left(x_{1}, x_{2}\right) d x_{2} d x_{1} \\
& +\int_{0}^{\infty} f_{3}(x, x) d x=1
\end{aligned}
$$

Let:

$$
I_{1}=\int_{0}^{\infty} \int_{0}^{x_{2}} f_{1}\left(x_{1}, x_{2}\right) d x_{1} d x_{2} \text { and } I_{2}=\int_{0}^{\infty} \int_{0}^{x_{1}} f_{2}\left(x_{1}, x_{2}\right) d x_{2} d x_{1}
$$

Then:

$$
\begin{aligned}
& I_{1}=\int_{0}^{\infty} \int_{0}^{x_{2}}\left(\alpha_{1}+\alpha_{3}\right)\left(a+b x_{1}+c x_{1}^{2}\right) e^{-\left(a x_{1}+\frac{b}{2} x_{1}^{2}+\frac{c}{3} x_{1}^{3}\right)} \\
& \times\left[1-e^{-\left(a x_{1}+\frac{b}{2} x_{1}^{2}+\frac{c}{3} x_{1}^{3}\right)}\right]^{\alpha_{1}+\alpha_{3}-1} \alpha_{2}\left(a+b x_{2}+c x_{2}^{2}\right) \\
& e^{-\left(a x_{2}+\frac{b}{2} x_{2}^{2}+\frac{c}{3} x_{2}^{3}\right)}\left[1-e^{-\left(a x_{2}+\frac{b}{2} x_{2}^{2}+\frac{c}{3} x_{2}^{3}\right)}\right]^{\alpha_{2}-1} d x_{1} d x_{2} \\
& =\int_{0}^{\infty} \alpha_{2}\left(a+b x_{2}+c x_{2}^{2}\right) e^{-\left(a x_{2}+\frac{b}{2} x_{2}^{2}+\frac{c}{3} x_{2}^{3}\right)} \\
& {\left[1-e^{-\left(a x_{2}+\frac{b}{2} x_{2}^{2}+\frac{c}{3} x_{2}^{3}\right)}\right]^{\alpha_{1}+\alpha_{2}+\alpha_{3}-1} d x_{2}}
\end{aligned}
$$

Similarly:

$$
\begin{aligned}
& I_{2}=\int_{0}^{\infty} \alpha_{1}\left(a+b x_{1}+c x_{1}^{2}\right) e^{-\left(a x_{1}+\frac{b}{2} x_{1}^{2}+\frac{c}{3} x_{1}^{3}\right)} \\
& {\left[1-e^{-\left(a x_{1}+\frac{b}{2} x_{1}^{2}+\frac{c}{3} x_{1}^{3}\right)}\right]^{\alpha_{1}+\alpha_{2}+\alpha_{3}-1} d x_{1} .}
\end{aligned}
$$

From Equation 12 and 13, we then get:

$$
\begin{aligned}
& \int_{0}^{\infty} f_{3}(x, x) d x=\int_{0}^{\infty}\left(\alpha_{1}+\alpha_{2}+\alpha_{3}\right)\left(a+b x+c x^{2}\right) e^{-\left(a x+\frac{b}{2} x^{2}+\frac{c}{3} x^{3}\right)} \\
& {\left[1-e^{-\left(a x+\frac{b}{2} x^{2}+\frac{c}{3} x^{3}\right)}\right]^{\alpha_{1}+\alpha_{2}+\alpha_{3}-1} d x-\int_{0}^{\infty} \alpha_{2}\left(a+b x+c x^{2}\right)} \\
& e^{-\left(a x+\frac{b}{2} x^{2}+\frac{c}{3} x^{3}\right)}\left[1-e^{-\left(a x+\frac{b}{2} x^{2}+\frac{c}{3} x^{3}\right)}\right]^{\alpha_{1}+\alpha_{2}+\alpha_{3}-1} d x \\
& -\int_{0}^{\infty} \alpha_{1}\left(a+b x_{1}+c x_{1}^{2}\right) e^{-\left(a x+\frac{b}{2} x^{2}+\frac{c}{3} x^{3}\right)}\left[1-e^{-\left(a x+\frac{b}{2} x^{2}+\frac{c}{3} x^{3}\right)}\right]^{\alpha_{1}+\alpha_{2}+\alpha_{3}-1} d x \\
& =\int_{0}^{\infty} \alpha_{3}\left(a+b x_{1}+c x_{1}^{2}\right) e^{-\left(a x+\frac{b}{2} x^{2}+\frac{c}{3} x^{3}\right)}\left[1-e^{-\left(a x+\frac{b}{2} x^{2}+\frac{c}{3} x^{3}\right)}\right]^{\alpha_{1}+\alpha_{2}+\alpha_{3}-1} d x .
\end{aligned}
$$

That is:

$$
f_{3}(x, x)
$$$$
=\alpha_{3}\left(a+b x_{1}+c x_{1}^{2}\right) e^{-\left(a x+\frac{b}{2} x^{2}+\frac{c}{3} x^{3}\right)}\left[1-e^{-\left(a x+\frac{b}{2} x^{2}+\frac{c}{3} x^{3}\right)}\right]^{\alpha_{1}+\alpha_{2}+\alpha_{3}-1}
$$

Hence the proof of the theorem is completed.

\section{Marginal Probability Density Function}

The following theorem gives the marginal PDF of $X_{i}$, $i=1,2$.

\section{Theorem 3}

The marginal probability density function of $X_{i}(i=1$, 2) can be derived as follows:

$$
\begin{aligned}
& f_{X_{i}}\left(x_{i}\right)=\left(\alpha_{i}+\alpha_{3}\right)\left(a+b x_{i}+c x_{i}^{2}\right) e^{-\left(a x_{i}+\frac{b}{2} x_{i}^{2}+\frac{c}{3} x_{i}^{3}\right)} \\
& {\left[1-e^{-\left(a x_{i}+\frac{b}{2} x_{i}^{2}+\frac{c}{3} x_{i}^{3}\right)}\right]^{\alpha_{i}+\alpha_{3}-1}}
\end{aligned}
$$

where, $i=1,2$.

\section{Proof}

Let $F_{X_{i}}\left(x_{i}\right)$ be the CDF of $X_{i}(i=1,2)$, given by:

$$
\begin{aligned}
& F_{X_{i}}\left(x_{i}\right)=P\left[X_{i} \leq x_{i}\right]=P\left[\max \left(U_{i}, U_{3}\right) \leq x_{i}\right] \\
& =P\left[U_{i} \leq x_{i}, U_{3} \leq x_{i}\right]
\end{aligned}
$$

Since $U_{i}$ and $U_{3}$ are independent, we have:

$$
F_{X_{i}}\left(x_{i}\right)=P\left[U_{i} \leq x_{i}\right] P\left[U_{3} \leq x_{i}\right]=\left[1-e^{-\left(a x_{i}+\frac{b}{2} x_{i}^{2}+\frac{c}{3} x_{i}^{3}\right)}\right]^{\alpha_{i}+\alpha_{3}}
$$

Differentiating with respect to $x_{i}$, we derive the formula given in Equation 14.

That is, $X_{i} \sim \operatorname{GQHR}\left(\alpha_{i}+\alpha_{3}, a, b, c\right)$. 


\section{Remark 4}

The relation between joint survival function, marginals and the joint $\mathrm{CDF}$ is given by:

$$
S_{X_{1}, X_{2}}\left(x_{1}, x_{2}\right)=1-F_{X_{1}}\left(x_{1}\right)-F_{X_{2}}\left(x_{2}\right)+F_{X_{1}, X_{2}}\left(x_{1}, x_{2}\right)
$$

Therefore, the joint survival function of $X_{1}$ and $X_{2}$ also can be derived.

\section{Lemma 5}

If $\left(X_{1}, X_{2}\right)$ have bivariate generalized quadratic hazard rate distribution, then $\max \left(X_{1}, X_{2}\right) \sim$ $\operatorname{GQHR}\left(\alpha_{1}+\alpha_{2}+\alpha_{3}, \mathrm{a}, \mathrm{b}, \mathrm{c}\right)$.

\section{Proof:}

$$
\begin{aligned}
& F_{\max }(x)=P\left[\max \left(X_{1}, X_{2}\right) \leq x\right]=P\left[X_{1} \leq x, X_{2} \leq x\right] \\
& =P\left[U_{1} \leq x, U_{2} \leq x, U_{3} \leq x\right] \\
& =\left[1-e^{-\left(a x+\frac{b}{2} x^{2}+\frac{c}{3} x^{3}\right)}\right]^{\alpha_{1}+\alpha_{2}+\alpha_{3}}
\end{aligned}
$$

That is the maximum of $X_{1}$ and $X_{2}$ is also generalized quadratic hazard rate.

\section{The Conditional Probability Density Function}

Since the marginal probability density function of $X_{i}$ $(i=1,2)$ is derived, we can now find the conditional probability density function.

\section{Theorem 6}

The conditional probability density function of $X_{i}$, given $X_{j}=x_{j}(i \neq j=1,2)$, is obtained by:

$$
f_{X_{i} \mid X_{j}}\left(x_{i} \mid x_{j}\right)=\left\{\begin{array}{l}
f_{X_{i} \mid X_{j}}^{(1)}\left(x_{i} \mid x_{j}\right) \text { if } x_{i}<x_{j} \\
f_{X_{i} \mid X_{j}}^{(2)}\left(x_{i} \mid x_{j}\right) \text { if } x_{j}<x_{i} \\
f_{X_{i} \mid X_{j}}^{(3)}\left(x_{i} \mid x_{j}\right) \text { if } x_{i}=x_{j} .
\end{array}\right.
$$

where:

$$
\begin{aligned}
& f_{X_{i} \mid X_{j}}^{(1)}\left(x_{i} \mid x_{j}\right)=\alpha_{j}\left(\alpha_{i}+\alpha_{3}\right)\left(a+b x_{i}+c x_{i}^{2}\right) e^{-\left(a x_{i}+\frac{b}{2} x_{i}^{2}+\frac{c}{3} x_{i}^{3}\right)} \\
& {\left[1-e^{-\left(a x_{i}+\frac{b}{2} x_{i}^{2}+\frac{c}{3} x_{i}^{3}\right)}\right]^{\alpha_{i}+\alpha_{3}-1}\left\{\left(\alpha_{j}+\alpha_{3}\right)\left[1-e^{-\left(a x_{j}+\frac{b}{2} x_{j}^{2}+\frac{c}{3} x_{j}^{3}\right)}\right]^{\alpha_{3}}\right\}^{-1},} \\
& f_{X_{i} \mid X_{j}}^{(2)}\left(x_{i} \mid x_{j}\right)=\alpha_{i}\left(a+b x_{i}+c x_{i}^{2}\right) e^{-\left(a x_{i}+\frac{b}{2} x_{i}^{2}+\frac{c}{3} x_{i}^{3}\right)} \\
& {\left[1-e^{-\left(a x_{i}+\frac{b}{2} x_{i}^{2}+\frac{c}{3} x_{i}^{3}\right)}\right]^{\alpha_{i}+-1},} \\
& f_{X_{i} \mid X_{j}}^{(3)}\left(x_{i} \mid x_{j}\right)=\frac{\alpha_{3}}{\alpha_{j}+\alpha_{3}}\left[1-e^{-\left(a x_{i}+\frac{b}{2} x_{i}^{2}+\frac{c}{3} x_{i}^{3}\right)}\right]^{\alpha_{i}}
\end{aligned}
$$

Proof

Since:

$$
f_{X_{i} \mid X_{j}}\left(x_{i} \mid x_{j}\right)=\frac{f_{X_{i}, X_{j}}\left(x_{i}, x_{j}\right)}{f_{X_{j}}\left(x_{j}\right)}
$$

By substituting from Equation 8 and 14, in the above relation, the theorem follows directly.

\section{Mathematical Expectations}

We can derive the $\mu_{r}^{\prime}$ ( $r$ th moments about zero) of $X_{i}$, $E\left[X_{1} X_{2}\right]$ and $E\left[X_{i} \mid X_{j}\right],(i \neq j=1,2)$, based on the results introduced in the last two subsections.

\section{Theorem 7}

The $r$ th moments of $X_{i}(i=1,2)$ about zero is given by:

$$
\begin{aligned}
& \mu_{r}^{\prime}=E\left[X_{i}^{r}\right]=\left(\alpha_{i}+\alpha_{3}\right) \sum_{j=0}^{\infty} \sum_{k=0}^{\infty} \sum_{\ell=0}^{\infty}(-1)^{j+k+\ell}\left(\begin{array}{c}
\alpha_{i}+\alpha_{3}-1 \\
j
\end{array}\right) \\
& \frac{(j+1)^{k+\ell} b^{k} c^{\ell}}{2^{k} 3^{\ell} k ! \ell !} \Gamma_{j, k, \ell}^{1},
\end{aligned}
$$

where:

$$
\begin{aligned}
& \Gamma_{j, k, \ell}^{1}=\frac{a \Gamma(2 k+3 \ell+r+1)}{[(j+1) a]^{2 k+3 \ell+r+1}}+\frac{b \Gamma(2 k+3 \ell+r+2)}{[(j+1) a]^{2 k+3 \ell+r+2}} \\
& +\frac{c \Gamma(2 k+3 \ell+r+3)}{[(j+1) a]^{2 k+3 \ell+r+3}} .
\end{aligned}
$$

Proof

Since the $r$ th moments is defined by:

$$
\mu_{r}^{\prime}=E\left[X_{i}^{r}\right]=\int_{0}^{\infty} x_{i}^{r} f_{X_{i}}\left(x_{i}\right) d x_{i}
$$

Substituting for $f_{X i}\left(x_{i}\right)$ from Equation 14, we get:

$$
\begin{aligned}
& E\left(X_{i}^{r}\right)=\left(\alpha_{i}+\alpha_{3}\right) \int_{0}^{\infty} x_{i}^{r}\left(a+b x_{i}+c x_{i}^{2}\right) e^{-\left(a x_{i}+\frac{b}{2} x_{i}^{2}+\frac{c}{3} x_{i}^{3}\right)} \\
& {\left[1-e^{-\left(a x_{i}+\frac{b}{2} x_{i}^{2}+\frac{c}{3} x_{i}^{3}\right)}\right]^{\alpha_{i}+\alpha_{3}-1} d x_{i}}
\end{aligned}
$$

Since $0<e^{-\left(a x_{i}+\frac{b}{2} x_{i}^{2}+\frac{c}{3} x_{i}^{3}\right)}<1$, then using the binomial expansion of $\left[1-e^{-\left(a x_{i}+\frac{b}{2} x_{i}^{2}+\frac{c}{3} x_{i}^{3}\right)}\right]^{\alpha_{i}+\alpha_{3}-1}$ given by:

$\left[1-e^{-\left(a x_{i}+\frac{b}{2} x_{i}^{2}+\frac{c}{3} x_{i}^{3}\right)}\right]^{\alpha_{i}+\alpha_{3}-1}=\sum_{j=0}^{\infty}(-1)^{j}\left(\begin{array}{c}\alpha_{i}+\alpha_{3}-1 \\ j\end{array}\right) e^{-j\left(a x_{i}+\frac{b}{2} x_{i}^{2}+\frac{c}{3} x_{i}^{3}\right)}$ 
Then:

$$
\begin{aligned}
& E\left(X_{i}^{r}\right)=\left(\alpha_{i}+\alpha_{3}\right) \sum_{j=0}^{\infty}(-1)^{j}\left(\begin{array}{c}
\alpha_{i}+\alpha_{3}-1 \\
j
\end{array}\right) \\
& \times \int_{0}^{\infty} x_{i}^{r}\left(a+b x_{i}+c x_{i}^{2}\right) e^{-(j+1)\left(a x_{i}+\frac{b}{2} x_{i}^{2}+\frac{c}{3} x_{i}^{3}\right)} d x_{i} .
\end{aligned}
$$

But the expansion of $e^{-(j+1) \frac{b}{2} x_{i}^{2}}$ and $e^{-(j+1) \frac{c}{3} x_{i}^{3}}$ are given by:

$$
\begin{aligned}
& e^{-(j+1) \frac{b}{2} x_{i}^{2}}=\sum_{k=0}^{\infty} \frac{(-1)^{k}(j+1)^{k} b^{k} x_{i}^{2 k}}{2^{k} k !} \text { and } \\
& e^{-(j+1) \frac{c}{3} x_{i}^{3}}=\sum_{\ell=0}^{\infty} \frac{(-1)^{\ell}(j+1)^{\ell} c^{\ell} x_{i}^{3 \ell}}{3^{\ell} \ell !},
\end{aligned}
$$

Substituting from Equation 21 into Equation 20, we have:

$$
\begin{aligned}
& E\left(X_{i}^{r}\right)=\left(\alpha_{i}+\alpha_{3}\right) \sum_{j=0}^{\infty} \sum_{k=0}^{\infty} \sum_{\ell=0}^{\infty}(-1)^{j+k+\ell}\left(\begin{array}{c}
\alpha_{i}+\alpha_{3}-1 \\
j
\end{array}\right) \\
& \times \frac{(j+1)^{k+\ell} b^{k} c^{\ell}}{2^{k} 3^{\ell} k ! \ell !} \int_{0}^{\infty}\left(a+b x_{i}+c x_{i}^{2}\right) x_{i}^{2 k+3 \ell+r} e^{-(j+1) a x_{i}} d x_{i} \\
& =\left(\alpha_{i}+\alpha_{3}\right) \sum_{j=0}^{\infty} \sum_{k=0}^{\infty} \sum_{\ell=0}^{\infty}(-1)^{j+k+\ell}\left(\begin{array}{c}
\alpha_{i}+\alpha_{3}-1 \\
j
\end{array}\right) \\
& \times \frac{(j+1)^{k+\ell} b^{k} c^{\ell}}{2^{k} 3^{\ell} k ! \ell !} \Gamma_{j, k, \ell}^{1},
\end{aligned}
$$

where:

$$
\Gamma_{j, k, \ell}^{1}=\int_{0}^{\infty}\left(a+b x_{i}+c x_{i}^{2}\right) x_{i}^{2 k+3 \ell+r} e^{-(j+1) a x_{i}} d x_{i}
$$

Let $(j+1) a x_{i}=u \Rightarrow x_{i}=\frac{u}{(j+1) a}$, , then:

$\Gamma_{j, k, \ell}^{1}=\frac{a \Gamma(2 k+3 \ell+r+1)}{[(j+1) a]^{2 k+3 \ell+r+1}}+\frac{b \Gamma(2 k+3 \ell+r+2)}{[(j+1) a]^{2 k+3 \ell+r+2}}$

$+\frac{c \Gamma(2 k+3 \ell+r+3)}{[(j+1) a]^{2 k+3 \ell+r+3}}$

where, $\Gamma(n)$, is the Gamma function.

Substituting from Equation 23 into Equation 22, we obtain the expression in Equation 19, which completes the proof.

\section{Theorem 8}

The expectation of $X_{1} X_{2}$ is given by:

$$
\begin{aligned}
& E\left[X_{1} X_{2}\right]=\sum_{i=0}^{\infty} \sum_{j=0}^{\infty} \sum_{k=0}^{\infty} \sum_{r=0}^{\infty} \sum_{\ell=0}^{\infty} \sum_{m=0}^{\infty} \sum_{n=0}^{\infty}\left[\alpha_{2}\left(\alpha_{1}+\alpha_{3}\right)\left(\begin{array}{c}
\alpha_{1}+\alpha_{3}-1 \\
i
\end{array}\right)\right. \\
& \left.\left(\begin{array}{c}
\alpha_{2}-1 \\
\ell
\end{array}\right)+\alpha_{1}\left(\alpha_{2}+\alpha_{3}\right)\left(\begin{array}{c}
\alpha_{2}+\alpha_{3}-1 \\
i
\end{array}\right)\left(\begin{array}{c}
\alpha_{1}-1 \\
\ell
\end{array}\right)\right] \beta_{i, j, k, \ell, m, n} \\
& +\alpha_{3} \sum_{i=0}^{\infty} \sum_{j=0}^{\infty} \sum_{k=0}^{\infty}\left(\begin{array}{c}
\alpha_{1}+\alpha_{2}+\alpha_{3}-1 \\
i
\end{array}\right) \Gamma_{i, j, k}^{2},
\end{aligned}
$$

where:

$$
\begin{aligned}
& \Gamma_{i, j, k}^{2}=(-1)^{i+j+k} \frac{(i+1)^{j+k} b^{j} c^{k}}{2^{j} 3^{k} j ! k !}\left[\frac{a \Gamma(2 j+3 k+3)}{[(i+1) a]^{2 j+3 k+3}}\right. \\
& +\frac{b \Gamma(2 j+3 k+4)}{\left.[(i+1) a]^{2 j+3 k+4}+\frac{c \Gamma(2 j+3 k+5)}{[(i+1) a]^{2 j+3 k+5}}\right],} \\
& \beta_{i, j, k, r, \ell, m, n}=(-1)^{i+j+k+r+\ell+m+n} \frac{(i+1)^{j+k+r}(\ell+1)^{m+n} a^{r} b^{j+m} c^{k+n}}{2^{j+m} 3^{k+n} j ! k ! r ! m ! n !} \\
& {\left[\frac{a^{2} \Gamma(p+4)}{\left(p_{1}+2\right)[(\ell+1) a]^{p+4}}+\frac{a b\left(2 p_{1}+5\right) \Gamma(p+5)}{\left(p_{1}+2\right)\left(p_{1}+3\right)[(\ell+1) a]^{p+5}}\right.} \\
& +\frac{a c\left(2 p_{1}+6\right) \Gamma(p+6)}{\left(p_{1}+2\right)\left(p_{1}+4\right)[(\ell+1) a]^{p+6}}+\frac{b^{2} \Gamma(p+6)}{\left(p_{1}+3\right)[(\ell+1) a]^{p+6}} \\
& \left.+\frac{b c\left(2 p_{1}+7\right) \Gamma(p+7)}{\left(p_{1}+3\right)\left(p_{1}+4\right)[(\ell+1) a]^{p+7}}+\frac{c^{2} \Gamma(p+8)}{\left(p_{1}+4\right)[(\ell+1) a]^{p+8}}\right] \\
& \text { and } p=p_{1}+2 m+3 n, \mathrm{p}_{1}=2 j+3 k+r .
\end{aligned}
$$

\section{Proof}

Since:

$$
\begin{aligned}
& E\left[X_{1} X_{2}\right]=\int_{0}^{\infty} \int_{0}^{\infty} x_{1} x_{2} f\left(x_{1}, x_{2}\right) d x_{1} d x_{2} \\
& =\int_{0}^{\infty} \int_{0}^{x_{2}} x_{1} x_{2} f_{1}\left(x_{1}, x_{2}\right) d x_{1} d x_{2}+\int_{0}^{\infty} \int_{0}^{x_{1}} x_{1} x_{2} f_{2}\left(x_{1}, x_{2}\right) d x_{2} d x_{1} \\
& +\int_{0}^{\infty} x^{2} f_{3}(x, x) d x_{1} d x_{2} .
\end{aligned}
$$

Let:

$$
\begin{aligned}
& I_{1}=\int_{0}^{\infty} \int_{0}^{x_{2}} x_{1} x_{2} f_{1}\left(x_{1}, x_{2}\right) d x_{1} d x_{2}, \\
& I_{2}=\int_{0}^{\infty} \int_{0}^{x_{1}} x_{1} x_{2} f_{2}\left(x_{1}, x_{2}\right) d x_{2} d x_{1} \\
& I_{3}=\int_{0}^{\infty} x^{2} f_{3}(x, x) d x_{1} d x_{2} .
\end{aligned}
$$

From Equation 9, we have:

$$
\begin{aligned}
& I_{1}=\left(\alpha_{1}+\alpha_{3}\right) \int_{0}^{\infty} \int_{0}^{x_{2}} x_{1} x_{2}\left(a+b x_{1}+c x_{1}^{2}\right) e^{-\left(a x_{1}+\frac{b}{2} x_{1}^{2}+\frac{c}{3} x_{1}^{3}\right)} \\
& {\left[1-e^{-\left(a x_{1}+\frac{b}{2} x_{1}^{2}+\frac{c}{3} x_{1}^{3}\right)}\right]^{\alpha_{1}+\alpha_{3}-1} \alpha_{2}\left(a+b x_{2}+c x_{2}^{2}\right) e^{-\left(a x_{2}+\frac{b}{2} x_{2}^{2}+\frac{c}{3} x_{2}^{3}\right)}} \\
& \times\left[1-e^{-\left(a x_{2}+\frac{b}{2} x_{2}^{2}+\frac{c}{3} x_{2}^{3}\right)}\right]^{\alpha_{2}-1} d x_{1} d x_{2}, \\
& \text { Since } 0<e^{-\left(a x_{2}+\frac{b}{2} x_{2}^{2}+\frac{c}{3} x_{2}^{3}\right)}<1, \text { by using the binomial } \\
& \text { expansion for }\left[1-e^{-\left(a x_{1}+\frac{b}{2} x_{1}^{2}+\frac{c}{3} x_{1}^{3}\right)}\right]^{\alpha_{1}+\alpha_{3}-1} \text {, we have: }
\end{aligned}
$$




$$
\begin{aligned}
& I_{1}=\left(\alpha_{1}+\alpha_{3}\right) \sum_{i=0}^{\infty}\left(\begin{array}{c}
\alpha_{1}+\alpha_{3}-1 \\
i
\end{array}\right)(-1)^{i} \int_{0}^{\infty} \int_{0}^{x_{2}} x_{1} x_{2}\left(a+b x_{1}+c x_{1}^{2}\right) e^{-(i+1)\left(a x_{1}+\frac{b}{2} x_{1}^{2}+\frac{c}{3} x_{1}^{3}\right)} \\
& \alpha_{2}\left(a+b x_{2}+c x_{2}^{2}\right) e^{-\left(a x_{2}+\frac{b}{2} x_{2}^{2}+\frac{c}{3} x_{2}^{3}\right)}\left[1-e^{-\left(a x_{2}+\frac{b}{2} x_{2}^{2}+\frac{c}{3} x_{2}^{3}\right)}\right]^{\alpha_{2}-1} d x_{1} d x_{2},
\end{aligned}
$$

By using the expansions of $e^{-(i+1) a x_{1}}, e^{-(i+1) \frac{b}{2} x_{1}^{2}}$ and $e^{-(i+1) \frac{c}{3} x_{1}^{3}}$, we have:

$$
\begin{aligned}
& I_{1}=\alpha_{2}\left(\alpha_{1}+\alpha_{3}\right) \sum_{i=0}^{\infty} \sum_{j=0}^{\infty} \sum_{k=0}^{\infty} \sum_{r=0}^{\infty}\left(\begin{array}{c}
\alpha_{1}+\alpha_{3}-1 \\
i
\end{array}\right)(-1)^{i+j+k+r} \\
& \frac{(i+1)^{j+k+r} b^{j} c^{k} a^{r}}{2^{j} 3^{k} j ! k ! r !} \int_{0}^{\infty} \int_{0}^{x_{2}} x_{1} x_{2}\left(a+b x_{1}+c x_{1}^{2}\right) x_{1}^{2 j+3 k+r} \\
& \left(a+b x_{2}+c x_{2}^{2}\right) e^{-\left(a x_{2}+\frac{b}{2} x_{2}^{2}+\frac{c}{3} x_{2}^{3}\right)}\left[1-e^{-\left(a x_{2}+\frac{b}{2} x_{2}^{2}+\frac{c}{3} x_{2}^{3}\right)}\right]^{\alpha_{2}-1} d x_{1} d x_{2} \\
& =\alpha_{2}\left(\alpha_{1}+\alpha_{3}\right) \sum_{i=0}^{\infty} \sum_{j=0}^{\infty} \sum_{k=0}^{\infty} \sum_{r=0}^{\infty}\left(\begin{array}{c}
\alpha_{1}+\alpha_{3}-1 \\
i
\end{array}\right)(-1)^{i+j+k+r} \frac{(i+1)^{j+k+r} b^{j} c^{k} a^{r}}{2^{j} 3^{k} j ! k ! r !} \\
& \int_{0}^{\infty} x_{2}\left[\frac{a x_{2}^{2 j+3 k+r+2}}{2 j+3 k+r+2}+\frac{b x_{2}^{2 j+3 k+r+3}}{2 j+3 k+r+3}+\frac{c x_{2}^{2 j+3 k+r+4}}{2 j+3 k+r+4}\right] \\
& \left(a+b x_{2}+c x_{2}^{2}\right) e^{-\left(a x_{2}+\frac{b}{2} x_{2}^{2}+\frac{c}{3} x_{2}^{3}\right)}\left[1-e^{-\left(a x_{2}+\frac{b}{2} x_{2}^{2}+\frac{c}{3} x_{2}^{3}\right)}\right]^{\alpha_{2}-1} d x_{2} \\
& =\alpha_{2}\left(\alpha_{1}+\alpha_{3}\right) \sum_{i=0}^{\infty} \sum_{j=0}^{\infty} \sum_{k=0}^{\infty} \sum_{r=0}^{\infty}\left(\alpha_{1}^{\alpha_{1}+\alpha_{3}-1} i_{i}\right)(-1)^{i+j+k+r} \\
& \frac{(i+1)^{j+k+r} b^{j} c^{k} a^{r}}{2^{j} 3^{k} j ! k ! r !} I_{11},
\end{aligned}
$$

where:

$$
\begin{aligned}
& I_{11}=\int_{0}^{\infty} x_{2}\left[\frac{a x_{2}^{2 j+3 k+r+2}}{2 j+3 k+r+2}+\frac{b x_{2}^{2 j+3 k+r+3}}{2 j+3 k+r+3}\right. \\
& \left.+\frac{c x_{2}^{2 j+3 k+r+4}}{2 j+3 k+r+4}\right]\left(a+b x_{2}+c x_{2}^{2}\right) \sum_{\ell=0}^{\infty}(-1)^{\ell}\left(\begin{array}{c}
\alpha_{2}-1 \\
\ell
\end{array}\right) e^{-(\ell+1)\left(a x_{2}+\frac{b}{2} x_{2}^{2}+\frac{c}{3} x_{2}^{3}\right)} d x_{2} \\
& =\sum_{\ell=0}^{\infty} \sum_{m=0}^{\infty} \sum_{n=0}^{\infty}(-1)^{\ell+m+n}\left(\begin{array}{c}
\alpha_{2}-1 \\
\ell
\end{array}\right) \frac{(\ell+1)^{m+n} b^{m} c^{n}}{2^{m} 3^{n} m ! n !} \int_{0}^{\infty}\left[\frac{a x_{2}^{2 j+3 k+r+3}}{2 j+3 k+r+2}\right. \\
& \left.+\frac{b x_{2}^{2 j+3 k+r+4}}{2 j+3 k+r+3}+\frac{c x_{2}^{2 j+3 k+r+5}}{2 j+3 k+r+4}\right] \\
& \left(a x_{2}^{2 m+3 n}+b x_{2}^{2 m+3 n+1}+c x_{2}^{2 m+3 n+2}\right) e^{-(\ell+1) a x_{2}} d x_{2} \\
& =\sum_{\ell=0}^{\infty} \sum_{m=0}^{\infty} \sum_{n=0}^{\infty}(-1)^{\ell+m+n}\left(\begin{array}{c}
\alpha_{2}-1 \\
l
\end{array}\right) \frac{(\ell+1)^{m+n} b^{m} c^{n}}{2^{m} 3^{n} m ! n !} \int_{0}^{\infty}\left[\frac{a^{2} x_{2}^{2 j+3 k+r+2 m+3 n+3}}{2 j+3 k+r+2}\right. \\
& +\frac{a b x_{2}^{2 j+3 k+r+2 m+3 n+4}}{2 j+3 k+r+3}+\frac{a c x_{2}^{2 j+3 k+r+2 m+3 n+5}}{2 j+3 k+r+4}+\frac{a b x_{2}^{2 j+3 k+r+2 m+3 n+4}}{2 j+3 k+r+2} \\
& +\frac{b^{2} x_{2}^{2 j+3 k+r+2 m+3 n+5}}{2 j+3 k+r+3}+\frac{c b x_{2}^{2 j+3 k+r+2 m+3 n+6}}{2 j+3 k+r+4}+\frac{a c x_{2}^{2 j+3 k+r+2 m+3 n+5}}{2 j+3 k+r+2} \\
& \left.+\frac{b c x_{2}^{2 j+3 k+r+2 m+3 n+6}}{2 j+3 k+r+3}+\frac{c^{2} x_{2}^{2 j+3 k+r+2 m+3 n+7}}{2 j+3 k+r+4}\right] e^{-(\ell+1) a x_{2}} d x_{2},
\end{aligned}
$$

Setting $p_{1}=2 j+3 k+r, p=p_{1}+2 m+3 n$ and $u=$ $(1+1) a x_{2}$, then:

$$
\begin{aligned}
& I_{11}=\sum_{l=0}^{\infty} \sum_{m=0}^{\infty} \sum_{n=0}^{\infty}(-1)^{l+m+n}\left(\begin{array}{c}
\alpha_{2}-1 \\
l
\end{array}\right) \frac{(l+1)^{m+n} b^{m} c^{n}}{2^{m} 3^{n} m ! n !} \\
& \int_{0}^{\infty}\left[\frac{a^{2} u^{p+3}}{\left(p_{1}+2\right)[(l+1) a]^{p+4}}+\frac{a b\left(2 p_{1}+5\right) u^{p+4}}{\left(p_{1}+2\right)\left(p_{1}+3\right)[(l+1) a]^{p+5}}\right. \\
& +\frac{a c\left(2 p_{1}+6\right) u^{p+5}}{\left(p_{1}+2\right)\left(p_{1}+4\right)[(\ell+1) a]^{p+6}}+\frac{b^{2} u^{p+5}}{\left(p_{1}+3\right)[(\ell+1) a]^{p+6}} \\
& \left.+\frac{c b\left(2 p_{1}+7\right) u^{p+6}}{\left(p_{1}+3\right)\left(p_{1}+4\right)[(\ell+1) a]^{p+7}}+\frac{c^{2} u^{p+7}}{\left(p_{1}+4\right)[(\ell+1) a]^{p+8}}\right] e^{-u} d u \\
& =\sum_{\ell=0}^{\infty} \sum_{m=0}^{\infty} \sum_{n=0}^{\infty}(-1)^{l+m+n}\left(\begin{array}{c}
\alpha_{2}-1 \\
\ell
\end{array}\right) \frac{(\ell+1)^{m+n} b^{m} c^{n}}{2^{m} 3^{n} m ! n !}\left[\frac{a^{2} \Gamma(p+4)}{\left(p_{1}+2\right)[(\ell+1) a]^{p+4}}\right. \\
& \frac{a b\left(2 p_{1}+5\right) \Gamma(p+5)}{\left(p_{1}+2\right)\left(p_{1}+3\right)[(\ell+1) a]^{p+5}}+\frac{a c\left(2 p_{1}+6\right) \Gamma(p+6)}{\left(p_{1}+2\right)\left(p_{1}+4\right)[(\ell+1) a]^{p+6}} \\
& +\frac{b^{2} \Gamma(p+6)}{\left(p_{1}+3\right)[(\ell+1) a]^{p+6}}+\frac{c b\left(2 p_{1}+7\right) \Gamma(p+7)}{\left(p_{1}+3\right)\left(p_{1}+4\right)[(\ell+1) a]^{p+7}} \\
& \left.+\frac{c^{2} \Gamma(p+8)}{\left(p_{1}+4\right)[(\ell+1) a]^{p+8}}\right] .
\end{aligned}
$$

Substituting from Equation 27 into Equation 26 we have:

$$
I_{1}=\left(\alpha_{1}+\alpha_{3}\right) \alpha_{2} \sum_{i=0}^{\infty} \sum_{j=0}^{\infty} \sum_{k=0}^{\infty} \sum_{r=0}^{\infty} \sum_{\ell=0}^{\infty} \sum_{m=0}^{\infty} \sum_{n=0}^{\infty}\left(\begin{array}{c}
\alpha_{1}+\alpha_{3}-1 \\
i
\end{array}\right)\left(\begin{array}{c}
\alpha_{2}-1 \\
\ell
\end{array}\right) \beta_{i, j, k, r, \ell, m, n},
$$

where:

$$
\begin{aligned}
& \beta_{i, j, k, r, l, m, n}=(-1)^{i+j+k+r+\ell+m+n} \frac{(i+1)^{j+k+r}(\ell+1)^{m+n} a^{r} b^{j+m} c^{k+n}}{2^{j+m} 3^{k+n} j ! k ! r ! m ! n !} \\
& {\left[\frac{a^{2} \Gamma(p+4)}{\left(p_{1}+2\right)[(\ell+1) a]^{p+4}}+\frac{a b\left(2 p_{1}+5\right) \Gamma(p+5)}{\left(p_{1}+2\right)\left(p_{1}+3\right)[(\ell+1) a]^{p+5}}\right.} \\
& +\frac{a c\left(2 p_{1}+6\right) \Gamma(p+6)}{\left(p_{1}+2\right)\left(p_{1}+4\right)[(\ell+1) a]^{p+6}}+\frac{b^{2} \Gamma(p+6)}{\left(p_{1}+3\right)[(\ell+1) a]^{p+6}} \\
& \left.\frac{c b\left(2 p_{1}+7\right) \Gamma(p+7)}{\left(p_{1}+3\right)\left(p_{1}+4\right)[(\ell+1) a]^{p+7}}+\frac{c^{2} \Gamma(p+8)}{\left(p_{1}+4\right)[(\ell+1) a]^{p+8}}\right] .
\end{aligned}
$$

From Equation 10, we can find $I_{2}$, by the same way:

$$
I_{2}=\left(\alpha_{2}+\alpha_{3}\right) \alpha_{1} \sum_{i=0}^{\infty} \sum_{j=0}^{\infty} \sum_{k=0}^{\infty} \sum_{r=0}^{\infty} \sum_{\ell=0}^{\infty} \sum_{m=0}^{\infty} \sum_{n=0}^{\infty}\left(\begin{array}{c}
\alpha_{2}+\alpha_{3}-1 \\
i
\end{array}\right)\left(\begin{array}{c}
\alpha_{1}-1 \\
\ell
\end{array}\right) \beta_{i, j, k, r, \ell, m, n}
$$

From Equation 11, we have: 


$$
\begin{aligned}
& I_{3}=\alpha_{3} \int_{0}^{\infty} x^{2}\left(a+b x+c x^{2}\right) e^{-\left(a x+\frac{b}{2} x^{2}+\frac{c}{3} x^{3}\right)} \\
& {\left[1-e^{-\left(a x+\frac{b}{2} x^{2}+\frac{c}{3} x^{3}\right)}\right]^{\alpha_{1}+\alpha_{2}+\alpha_{3}-1} d x}
\end{aligned}
$$

Since $0<e^{-\left(a x+\frac{b}{2} x^{2}+\frac{c}{3} x^{3}\right)}<1$, by using the binomial expansion for $\left[1-e^{-\left(a x+\frac{b}{2} x^{2}+\frac{c}{3} x^{3}\right)}\right]^{\alpha_{1}+\alpha_{2}+\alpha_{3}-1}$, we have:

$$
I_{3}=\alpha_{3} \sum_{i=0}^{\infty}\left(\begin{array}{c}
\alpha_{1}+\alpha_{2}+\alpha_{3}-1 \\
i
\end{array}\right)(-1)^{i} \int_{0}^{\infty} x^{2}\left(a+b x+c x^{2}\right) e^{-(i+1)\left(a x+\frac{b}{2} x^{2}+\frac{c}{3} x^{3}\right)} d x
$$

By using the Taylor's expression for $e^{-(i+1) \frac{b}{2} x^{2}}$ and $e^{-(i+1) \frac{c}{3} x^{3}}$, we have:

$$
\begin{aligned}
& I_{3}=\alpha_{3} \sum_{i=0}^{\infty} \sum_{j=0}^{\infty} \sum_{k=0}^{\infty}\left(\begin{array}{c}
\alpha_{1}+\alpha_{2}+\alpha_{3}-1 \\
i
\end{array}\right)(-1)^{i+j+k} \frac{(i+1)^{j+k} b^{j} c^{k}}{2^{j} 3^{k} j ! k !} \times \mathbb{S} \\
& \int_{0}^{\infty}\left(a x^{2}+b x^{3}+c x^{4}\right) x^{2 j+3 k} e^{-(i+1) a x} d x
\end{aligned}
$$

Put $u=(I+1) a x$, we have:

$$
\begin{aligned}
& I_{3}=\alpha_{3} \sum_{i=0}^{\infty} \sum_{j=0}^{\infty} \sum_{k=0}^{\infty}\left(\begin{array}{c}
\alpha_{1}+\alpha_{2}+\alpha_{3}-1 \\
i
\end{array}\right)(-1)^{i+j+k} \frac{(i+1)^{j+k} b^{j} c^{k}}{2^{j} 3^{k} j ! k !} \\
& \times \int_{0}^{\infty}\left[\begin{array}{l}
\frac{a u^{2 j+3 k+2}}{[(i+1) a]^{2 j+3 k+3}}+\frac{b u^{2 j+3 k+3}}{[(i+1) a]^{2 j+3 k+4}} \\
+\frac{c u^{2 j+3 k+4}}{[(i+1) a]^{2 j+3 k+5}}
\end{array}\right] e^{-u} d u \\
& =\alpha_{3} \sum_{i=0}^{\infty} \sum_{j=0}^{\infty} \sum_{k=0}^{\infty}\left(\begin{array}{c}
\alpha_{1}+\alpha_{2}+\alpha_{3}-1 \\
i
\end{array}\right) \Gamma_{i, j, k}^{2},
\end{aligned}
$$

where:

$$
\begin{aligned}
& \Gamma_{i, j, k}^{2}=(-1)^{i+j+k} \frac{(i+1)^{j+k} b^{j} c^{k}}{2^{j} 3^{k} j ! k !}\left[\frac{a \Gamma(2 j+3 k+3)}{[(i+1) a]^{2 j+3 k+3}}\right. \\
& \left.+\frac{b \Gamma(2 j+3 k+4)}{[(i+1) a]^{2 j+3 k+4}}+\frac{c \Gamma(2 j+3 k+5)}{[(i+1) a]^{2 j+3 k+5}}\right] .
\end{aligned}
$$

Substituting from Equation 28-30 into Equation 25, we have Equation 24, this completes the proof.

From Theorem 7, setting $r=1, i=1,2$ and Theorem 8 , we can find the covariance between $X_{1}, X_{2}$ :

$$
\operatorname{Cov}\left(X_{1}, X_{2}\right)=E\left[X_{1} X_{2}\right]-E\left[X_{1}\right] E\left[X_{2}\right]
$$

and:

$$
\operatorname{Var}\left(X_{i}\right)=E\left[X_{i}^{2}\right]-\left(E\left[X_{i}\right]\right)^{2} .
$$

Also, we can compute the correlation coefficient between $X_{1}, X_{2}$ as follows:

$$
\rho_{X_{1}, X_{2}}=\frac{\operatorname{Cov}\left(X_{1}, X_{2}\right)}{\sqrt{\operatorname{Var}\left(X_{1}\right) \operatorname{Var}\left(X_{2}\right)}} .
$$

\section{Theorem 9}

The conditional expectation of $X_{i}$, given $X_{j}=x_{j}(i \neq j$ $=1,2)$ is given by:

$$
\begin{aligned}
& E\left[X_{i} \mid X_{j}=x_{j}\right]=\sum_{k=0}^{\infty} \sum_{\ell=0}^{\infty} \sum_{m=0}^{\infty}(-1)^{k+\ell+m} \frac{b^{\ell} c^{m}}{2^{\ell} 3^{m} \ell ! m !}\left\{W_{i, j}\left(x_{j}\right)\right. \\
& \times\left(\begin{array}{c}
\alpha_{i}+\alpha_{3}-1 \\
k
\end{array}\right)(k+1)^{\ell+m} I_{x_{j}}^{(1)}(k, \ell, m)+\alpha_{i}\left(\begin{array}{c}
\alpha_{i}-1 \\
k
\end{array}\right)(k+1)^{m+\ell} \\
& \left.\times I_{x_{j}}^{(2)}(k, \ell, m)+\frac{\alpha_{3}}{\alpha_{j}+\alpha_{3}}\left(\begin{array}{c}
\alpha_{i} \\
k
\end{array}\right) k^{\ell+m} \frac{\Gamma(2 \ell+3 m+2)}{(k a)^{2 \ell+3 m+2}}\right\},
\end{aligned}
$$

where:

$$
\begin{aligned}
& W_{i, j}\left(x_{j}\right)=\frac{\alpha_{j}\left(\alpha_{i}+\alpha_{3}\right)}{\alpha_{j}+\alpha_{3}}\left[1-e^{-\left(a x_{j}+\frac{b}{2} x_{j}^{2}+\frac{c}{3} x_{j}^{3}\right)}\right]^{\alpha_{3}}, \\
& I_{x_{j}}^{(1)}(k, \ell, m)=\int_{0}^{(k+1) a x_{j}} J_{k, \ell, m}(u) d u, \\
& I_{x_{j}}^{(2)}(k, \ell, m)=\int_{(k+1) a x_{j}}^{\infty} J_{k, \ell, m}(u) d u, \\
& J_{k, \ell, m}(u)=\left[\frac{a u^{2 \ell+3 m+1}}{[(k+1) a]^{2 \ell+3 m+2}}+\frac{b u^{2 \ell+3 m+2}}{[(k+1) a]^{2 \ell+3 m+3}}\right. \\
& \left.+\frac{c u^{2 \ell+3 m+3}}{[(k+1) a]^{2 \ell+3 m+4}}\right] e^{-u} .
\end{aligned}
$$

\section{Proof}

Starting with:

$$
\begin{aligned}
& E\left[X_{i} \mid X_{j}=x_{j}\right]=\int_{0}^{\infty} x_{i} f_{X_{i} \mid X_{j}}\left(x_{i} \mid x_{j}\right) d x_{i} \\
& =\int_{0}^{x_{j}} x_{i} f_{X_{i} \mid X_{j}}^{(1)}\left(x_{i} \mid x_{j}\right) d x_{i}+\int_{x_{j}}^{\infty} x_{i} f_{X_{i} \mid X_{j}}^{(2)}\left(x_{i} \mid x_{j}\right) d x_{i} \\
& +\int_{0}^{\infty} x_{i} f_{X_{i} \mid X_{j}}^{(3)}\left(x_{i} \mid x_{j}\right) d x_{i} .
\end{aligned}
$$

Substituting from Equation 17 into Equation 32, we obtain Equation 31.

\section{Moment Generating Functions}

In this section, we introduce the joint moment generating function of $\left(X_{1}, X_{2}\right)$. The marginal moment generating function of $X_{i}(i=1,2)$ also derived.

\section{Lemma 10}

The moment generating function of $X_{i}(i=1,2)$ is given as follows: 


$$
\begin{aligned}
& M_{X_{i}}(t)=\left(\alpha_{i}+\alpha_{3}\right) \sum_{j=0}^{\infty} \sum_{k=0}^{\infty} \sum_{l=0}^{\infty}\left(\begin{array}{c}
\alpha_{i}+\alpha_{3}-1 \\
j
\end{array}\right) \frac{(-1)^{j+k+l}(j+1)^{k+l} b^{k} c^{l}}{2^{k} 3^{l} k ! l !} \\
& \times\left[\frac{a \Gamma(2 k+3 \ell+1)}{[(j+1) a+t]^{2 k+3 \ell+1}}+\frac{b \Gamma(2 k+3 \ell+2)}{[(j+1) a+t]^{2 k+3 \ell+2}}\right. \\
& \left.+\frac{c \Gamma(2 k+3 \ell+3)}{[(j+1) a+t]^{2 k+3 \ell+3}}\right] .
\end{aligned}
$$

\section{Proof}

Since:

$$
M_{X_{i}}(t)=E\left[e^{-t X_{i}}\right]=\int_{0}^{\infty} e^{-t x_{i}} f_{X_{i}}\left(x_{i}\right) d x_{i},
$$

and substituting for $f_{X i}\left(x_{i}\right)$ from Equation 14, we get:

$$
\begin{aligned}
& M_{X_{i}}(t)=\left(\alpha_{i}+\alpha_{3}\right) \int_{0}^{\infty} e^{-t x_{i}}\left(a+b x_{i}+c x_{i}^{2}\right) e^{-\left(a x_{i}+\frac{b}{2} x_{i}^{2}+\frac{c}{3} x_{i}^{3}\right)} \\
& {\left[1-e^{-\left(a x_{i}+\frac{b}{2} x_{i}^{2}+\frac{c}{3} x_{i}^{3}\right)}\right]^{\alpha_{i}+\alpha_{3}-1} d x_{i}}
\end{aligned}
$$

From which we can derive the expression given in Equation 33.

\section{Remark 11}

The moment generating function $M_{X i}(t)$ can be used to derive the marginal expectation of $\mathrm{X}_{\mathrm{i}}$ as follows:

$$
\mu_{r}^{\prime}=E\left[X^{r}\right]=(-1)^{r}\left[\frac{d^{r}}{d t^{r}} M_{X_{i}}(t)\right]_{t=0} .
$$

From Equation 33, we obtain:

$$
\begin{aligned}
& \frac{d^{r}}{d t_{i}^{r}} M_{X_{i}}(t)=(-1)^{r}\left(\alpha_{i}+\alpha_{3}\right) \sum_{j=0}^{\infty} \sum_{k=0}^{\infty} \sum_{l=0}^{\infty}\left(\begin{array}{c}
\alpha_{i}+\alpha_{3}-1 \\
j
\end{array}\right)(-1)^{j+k+l} \\
& \times \frac{(j+1)^{k+l} b^{k} c^{l}}{2^{k} 3^{l} k ! l !}\left[\frac{a \Gamma(2 k+3 \ell+r+1)}{[(j+1) a+t]^{2 k+3 \ell+r+1}}+\frac{b \Gamma(2 k+3 \ell+r+2)}{[(j+1) a+t]^{2 k+3 \ell+r+2}}\right. \\
& \left.+\frac{c \Gamma(2 k+3 \ell+r+3)}{[(j+1) a+t]^{2 k+3 \ell+r+3}}\right]
\end{aligned}
$$

Therefore:

$$
\begin{aligned}
& {\left[\frac{d^{r}}{d t_{i}^{r}} M_{X_{i}}(t)\right]_{t=0}=(-1)^{r}\left(\alpha_{i}+\alpha_{3}\right) \sum_{j=0}^{\infty} \sum_{k=0}^{\infty} \sum_{l=0}^{\infty}\left(\begin{array}{c}
\alpha_{i}+\alpha_{3}-1 \\
j
\end{array}\right)(-1)^{j+k+l}} \\
& \times \frac{(j+1)^{k+l} b^{k} c^{l}}{2^{k} 3^{l} k ! l !}\left[\frac{a \Gamma(2 k+3 l+r+1)}{[(j+1) a]^{2 k+3 l+r+1}}+\frac{b \Gamma(2 k+3 l+r+2)}{[(j+1) a]^{2 k+3 l+r+2}}\right. \\
& \left.+\frac{c \Gamma(2 k+3 l+r+3)}{[(j+1) a]^{2 k+3 l+r+3}}\right] .
\end{aligned}
$$

Substituting from Equation 35 into Equation 34, we obtain $\mu_{r}^{\prime}$ as given in Equation 19.

\section{Theorem 12}

The joint moment generating function of $\left(X_{1}, X_{2}\right)$ is given as follows:

$$
\begin{aligned}
& M_{X_{1}, X_{2}}\left(t_{1}, t_{2}\right) \\
& =\sum_{i=0}^{\infty} \sum_{j=0}^{\infty} \sum_{k=0}^{\infty} \sum_{r=0}^{\infty} \sum_{l=0}^{\infty} \sum_{m=0}^{\infty} \sum_{n=0}^{\infty}\left\{\alpha_{2}\left(\alpha_{1}+\alpha_{3}\right)\left(\begin{array}{c}
\alpha_{1}+\alpha_{3}-1 \\
i
\end{array}\right)\left(\begin{array}{c}
\alpha_{2}-1 \\
\ell
\end{array}\right)\right. \\
& \times\left[(i+1) a+t_{1}\right]^{r} F\left(\Gamma, t_{2}\right) \\
& +\alpha_{1}\left(\alpha_{2}+\alpha_{3}\right)\left(\begin{array}{c}
\alpha_{2}+\alpha_{3}-1 \\
i
\end{array}\right)\left(\begin{array}{c}
\alpha_{1}-1 \\
\ell
\end{array}\right)\left[(i+1) a+t_{2}\right]^{r} \\
& \left.F\left(\Gamma, t_{1}\right)\right\}+\alpha_{3} \sum_{i=0}^{\infty} \sum_{j=0}^{\infty} \sum_{k=0}^{\infty}\left(\begin{array}{c}
\alpha_{1}+\alpha_{2}+\alpha_{3}-1 \\
i
\end{array}\right) F\left(\Gamma, t_{1}, t_{2}\right),
\end{aligned}
$$

where:

$$
\begin{aligned}
& F\left(\Gamma, t_{i}\right)=(-1)^{i+j+k+r+\ell+m+n} \frac{(i+1)^{j+k}(\ell+1)^{m+n} b^{j+m} c^{k+n}}{2^{j+m} 3^{k+n} j ! k ! r ! m ! n !} \\
& {\left[\frac{a^{2} \Gamma(p+2)}{\left(p_{1}+1\right)\left[(\ell+1) a+t_{i}\right]^{p+2}}+\frac{a b\left(2 p_{1}+3\right) \Gamma(p+3)}{\left(p_{1}+1\right)\left(p_{1}+2\right)\left[(\ell+1) a+t_{i}\right]^{p+3}}\right.} \\
& +\frac{a c\left(2 p_{1}+4\right) \Gamma(p+4)}{\left(p_{1}+1\right)\left(p_{1}+3\right)\left[(\ell+1) a+t_{i}\right]^{p+4}}+\frac{b^{2} \Gamma(p+4)}{\left(p_{1}+2\right)\left[(\ell+1) a+t_{i}\right]^{p+4}} \\
& \left.+\frac{b c\left(2 p_{1}+5\right) \Gamma(p+5)}{\left(p_{1}+2\right)\left(p_{1}+3\right)\left[(\ell+1) a+t_{i}\right]^{p+5}}+\frac{c^{2} \Gamma(p+6)}{\left(p_{1}+3\right)\left[(\ell+1) a+t_{i}\right]^{p+6}}\right], \\
& i=1,2 . \\
& F\left(\Gamma, t_{1}, t_{2}\right)=(-1)^{i+j+k} \frac{(i+1)^{j+k} b^{j} c^{k}}{2^{j} 3^{k} j ! k !}\left[\frac{a \Gamma(2 j+3 m+1)}{\left[(i+1) a+t_{1}+t_{2}\right]^{2 j+3 m+1}}\right. \\
& \left.+\frac{b \Gamma(2 j+3 m+2)}{\left[(i+1) a+t_{1}+t_{2}\right]^{2 j+3 m+2}}+\frac{c \Gamma(2 j+3 m+3)}{\left[(i+1) a+t_{1}+t_{2}\right]^{2 j+3 m+3}}\right], \\
& \quad p=2 m+3 n+p_{1}, p_{1}=2 j+3 m+r .
\end{aligned}
$$

\section{Proof}

Since:

$$
\begin{aligned}
& M\left(t_{1}, t_{2}\right)=E\left[e^{-\left(t_{1} X_{1}+t_{2} X_{2}\right)}\right]=\int_{0}^{\infty} \int_{0}^{\infty} e^{-\left(t_{1} x_{1}+t_{2} x_{2}\right)} f\left(x_{1}, x_{2}\right) d x_{1} d x_{2} \\
& =\int_{0}^{\infty} \int_{0}^{x_{2}} e^{-\left(t_{1} x_{1}+t_{2} x_{2}\right)} f_{1}\left(x_{1}, x_{2}\right) d x_{1} d x_{2} \\
& +\int_{0}^{\infty} \int_{0}^{x_{1}} e^{-\left(t_{1} x_{1}+t_{2} x_{2}\right)} f_{2}\left(x_{1}, x_{2}\right) d x_{2} d x_{1}+\int_{0}^{\infty} e^{-\left(t_{1}+t_{2}\right) x} f_{3}(x, x) d x .
\end{aligned}
$$

Let:

$$
\begin{aligned}
& I_{1}=\int_{0}^{\infty} \int_{0}^{x_{2}} e^{-\left(t_{1} x_{1}+t_{2} x_{2}\right)} f_{1}\left(x_{1}, x_{2}\right) d x_{1} d x_{2}, \\
& I_{2}=\int_{0}^{\infty} \int_{0}^{x_{1}} e^{-\left(t_{1} x_{1}+t_{2} x_{2}\right)} f_{2}\left(x_{1}, x_{2}\right) d x_{2} d x_{1}, \\
& I_{3}=\int_{0}^{\infty} e^{-\left(t_{1}+t_{2}\right) x} f_{3}(x, x) d x .
\end{aligned}
$$

From Equation 9, we have: 


$$
\begin{aligned}
& I_{1}=\left(\alpha_{1}+\alpha_{3}\right) \int_{0}^{\infty} \int_{0}^{x_{2}} e^{-\left(t_{1} x_{1}+t_{2} x_{2}\right)}\left(a+b x_{1}+c x_{1}^{2}\right) e^{-\left(a \alpha_{1}+\frac{b}{2} x_{1}^{2}+\frac{c}{3} x_{1}^{3}\right)} \\
& {\left[1-e^{-\left(a x_{1}+\frac{b}{2} x_{1}^{2}+\frac{c}{3} x_{1}^{3}\right)}\right)^{\alpha_{1}+\alpha_{3}-1} \alpha_{2}\left(a+b x_{2}+c x_{2}^{2}\right) e^{-\left(a x_{2}+\frac{b}{2} x_{2}^{2}+\frac{c}{3} x_{2}^{3}\right)}} \\
& {\left[1-e^{-\left(a x_{2}+\frac{b}{2} x_{2}^{2}+\frac{c}{3} x_{2}\right)}\right]^{\alpha_{2}-1} d x_{1} d x_{2} .}
\end{aligned}
$$

Since $0<e^{-\left(a x_{2}+\frac{b}{2} x_{2}^{2}+\frac{c}{3} x_{2}^{3}\right)}<1$, using the binomial expansion for $\left[1-e^{-\left(a \alpha_{1}+\frac{b}{2} x^{2}+\frac{c}{3} x_{1}^{3}\right)}\right]^{\alpha_{1}+\alpha_{3}-1}$, we get:

$$
\begin{aligned}
& I_{1}=\left(\alpha_{1}+\alpha_{3}\right) \sum_{i=0}^{\infty}\left(\alpha_{1}+\alpha_{3}-1\right)(-1)^{i} \int_{0}^{\infty} \int_{0}^{x_{2}} e^{-\left(t x_{1}+t_{1}+x_{2}\right)}\left(a+b x_{1}+c x_{1}^{2}\right) \\
& \times e^{-(i+1)\left(a x_{1}+\frac{b}{2} x_{1}^{2}+\frac{c}{3} x_{1}^{3}\right)} \alpha_{2}\left(a+b x_{2}+c x_{2}^{2}\right) e^{-\left(a x_{2}+\frac{b}{2} x_{2}^{2}+\frac{c}{3} x_{2}^{3}\right)} \\
& {\left[1-e^{-\left(a x_{2}+\frac{b}{2} x_{2}^{2}+\frac{c}{3} x_{2}^{3}\right)}\right]^{\alpha_{2}-1} d x_{1} d x_{2}}
\end{aligned}
$$

Using the Taylor's expression for $e^{-(i+1) \frac{b}{2} x_{1}^{2}}$, $e^{-(i+1) \frac{c}{3} x_{1}^{3}}$ and $e^{-\left[(i+1) a+t_{1}\right] x_{1}}$, we have:

$$
I_{1}
$$$$
=\alpha_{2}\left(\alpha_{1}+\alpha_{3}\right) \sum_{i=0}^{\infty} \sum_{j=0}^{\infty} \sum_{k=0}^{\infty} \sum_{r=0}^{\infty}\left({ }^{\alpha_{1}+\alpha_{3}-1}{ }_{i}\right)(-1)^{i+j+k+r} \frac{(i+1)^{j+k}}{2^{j} 3^{k} j ! k ! r !}
$$$$
\times\left[(i+1) a+t_{1}\right]^{r} b^{j} c^{k} \int_{0}^{\infty} \int_{0}^{x_{2}} e^{-t_{2} x_{2}}\left(a+b x_{1}+c x_{1}^{2}\right) x_{1}^{2 j+3 k+r}
$$$$
\left(a+b x_{2}+c x_{2}^{2}\right) e^{-\left(a x_{2}+\frac{b}{2} x_{2}^{2}+\frac{c}{3} x_{2}^{3}\right)}\left[1-e^{-\left(a x_{2}+\frac{b}{2} x_{2}+\frac{c}{3} x_{2}^{3}\right)}\right]^{\alpha_{2}-1} d x_{1} d x_{2}
$$$$
=\alpha_{2}\left(\alpha_{1}+\alpha_{3}\right) \sum_{i=0}^{\infty} \sum_{j=0}^{\infty} \sum_{k=0}^{\infty} \sum_{r=0}^{\infty}\left({ }^{\alpha_{1}+\alpha_{3}-1}{ }_{i}\right)(-1)^{i+j+k+r} \frac{(i+1)^{j+k}}{2^{j} 3^{k} j ! k ! r !}
$$$$
\times\left[(i+1) a+t_{1}\right]^{r} b^{j} c^{k} \int_{0}^{\infty} e^{-t_{2} x_{2}}\left[\begin{array}{l}
\frac{a x_{2}^{2 j+3 k+r+1}}{2 j+3 k+r+1} \\
+\frac{b x_{2}^{2 j+3 k+r+2}}{2 j+3 k+r+2}
\end{array}\right.
$$$$
\left.+\frac{c x_{2}^{2 j+3 k+r+3}}{2 j+3 k+r+3}\right]\left(a+b x_{2}+c x_{2}^{2}\right) e^{-\left(a x_{2}+\frac{b}{2} x_{2}^{2}+\frac{c}{3} x_{2}^{3}\right)}
$$$$
\left[1-e^{-\left(a x_{2}+\frac{b}{2} x_{2}^{2}+\frac{c}{3} x_{2}\right)}\right]^{\alpha_{2}-1} d x_{2}
$$$$
=\alpha_{2}\left(\alpha_{1}+\alpha_{3}\right) \sum_{i=0}^{\infty} \sum_{j=0}^{\infty} \sum_{k=0}^{\infty} \sum_{r=0}^{\infty}\left({ }^{\alpha_{1}+\alpha_{3}-1}\right)(-1)^{i+j+k+r} \frac{(i+1)^{j+k}}{2^{j} 3^{k} j ! k ! r !}
$$

$$
\times\left[(i+1) a+t_{1}\right]^{r} b^{j} c^{k} I_{11},
$$

where:

$$
\begin{aligned}
& I_{11}=\int_{0}^{\infty} e^{-t_{2} x_{2}}\left[\frac{a x_{2}^{2 j+3 k+r+1}}{2 j+3 k+r+1}+\frac{b x_{2}^{2 j+3 k+r+2}}{2 j+3 k+r+2}\right. \\
& \left.+\frac{c x_{2}^{2 j+3 k+r+3}}{2 j+3 k+r+3}\right]\left(a+b x_{2}+c x_{2}^{2}\right) e^{-\left(a x_{2}+\frac{b}{2} x_{2}^{2}+\frac{c}{3} x_{2}^{3}\right)} \\
& \times\left[1-e^{-\left(a_{2}+\frac{b}{2} x_{2}^{2}+\frac{c}{3} x_{2}\right)}\right]^{\alpha_{2}-1} d x_{2} \\
& =\sum_{\ell=0}^{\infty} \sum_{m=0}^{\infty} \sum_{n=0}^{\infty}(-1)^{\ell+m+n}\left(\begin{array}{c}
\alpha_{2}-1 \\
\ell
\end{array}\right) \frac{(\ell+1)^{m+n} b^{m} c^{n}}{2^{m} 3^{n} m ! n !} \int_{0}^{\infty}\left[\frac{a x_{2}^{2 j+3 k+r+1}}{2 j+3 k+r+1}\right. \\
& \left.+\frac{b x_{2}^{2 j+3 k+r+2}}{2 j+3 k+r+2}+\frac{c x_{2}^{2 j+3 k+r+3}}{2 j+3 k+r+3}\right] \\
& \times\left(a x_{2}^{2 m+3 n}+b x_{2}^{2 m+3 n+1}+c x_{2}^{2 m+3 n+2}\right) e^{-\left[(l+1) a+t_{2}\right] x_{2}} d x_{2} \\
& =\sum_{\ell=0}^{\infty} \sum_{m=0}^{\infty} \sum_{n=0}^{\infty}(-1)^{l+m+n}\left(\begin{array}{c}
\alpha_{2}-1 \\
l
\end{array}\right) \frac{(\ell+1)^{m+n} b^{m} c^{n}}{2^{m} 3^{n} m ! n !} \\
& \times \int_{0}^{\infty}\left[\frac{a^{2} x_{2}^{2 j+3 k+r+2 m+3 n+1}}{2 j+3 k+r+1}+\frac{a b x_{2}^{2 j+3 k+r+2 m+3 n+2}}{2 j+3 k+r+2}\right. \\
& +\frac{a c x_{2}^{2 j+3 k+r+2 m+3 n+3}}{2 j+3 k+r+3}+\frac{a b x_{2}^{2 j+3 k+r+2 m+3 n+2}}{2 j+3 k+r+1} \\
& +\frac{b^{2} x_{2}^{2 j+3 k+r+2 m+3 n+3}}{2 j+3 k+r+2}+\frac{c b x_{2}^{2 j+3 k+r+2 m+3 n+4}}{2 j+3 k+r+3} \\
& +\frac{a c x_{2}^{2 j+3 k+r+2 m+3 n+3}}{2 j+3 k+r+1}+\frac{b c x_{2}^{2 j+3 k+r+2 m+3 n+4}}{2 j+3 k+r+2} \\
& \left.+\frac{c^{2} x_{2}^{2 j+3 k+r+2 m+3 n+5}}{2 j+3 k+r+3}\right] e^{-\left[(\ell+1) a+t_{2}\right] x_{2}} d x_{2},
\end{aligned}
$$

Setting $p_{1}=2 j+3 k+r$ and $p=p_{1}+2 m+3 n$, $u=\left[(\ell+1) a+t_{2}\right] x_{2}$, we have:

$$
\begin{aligned}
& I_{11}=\sum_{\ell=0}^{\infty} \sum_{m=0}^{\infty} \sum_{n=0}^{\infty}(-1)^{\ell+m+n}\left(\begin{array}{c}
\alpha_{2}-1 \\
\ell
\end{array}\right) \frac{(\ell+1)^{m+n} b^{m} c^{n}}{2^{m} 3^{n} m ! n !} \\
& \times\left[\frac{a^{2} \Gamma(p+2)}{\left(p_{1}+1\right)\left[(\ell+1) a+t_{2}\right]^{p+2}}+\frac{a b\left(2 p_{1}+3\right) \Gamma(p+3)}{\left(p_{1}+1\right)\left(p_{1}+2\right)\left[(\ell+1) a+t_{2}\right]^{p+3}}\right. \\
& +\frac{a c\left(2 p_{1}+4\right) \Gamma(p+4)}{\left(p_{1}+1\right)\left(p_{1}+3\right)\left[(\ell+1) a+t_{2}\right]^{p+4}}+\frac{b^{2} \Gamma(p+4)}{\left(p_{1}+2\right)\left[(\ell+1) a+t_{2}\right]^{p+4}} \\
& +\frac{c b\left(2 p_{1}+5\right) \Gamma(p+5)}{\left(p_{1}+2\right)\left(p_{1}+3\right)\left[(\ell+1) a+t_{2}\right]^{p+5}} \\
& \left.+\frac{c^{2} \Gamma(p+6)}{\left(p_{1}+3\right)\left[(\ell+1) a+t_{2}\right]^{p+6}}\right] .
\end{aligned}
$$

Substituting from Equation 39 into Equation 38 we have:

$$
\begin{aligned}
& I_{1}=\alpha_{2}\left(\alpha_{1}+\alpha_{3}\right) \sum_{i=0}^{\infty} \sum_{j=0}^{\infty} \sum_{k=0}^{\infty} \sum_{r=0}^{\infty} \sum_{l=0}^{\infty} \sum_{m=0}^{\infty} \sum_{n=0}^{\infty}\left(\begin{array}{c}
\alpha_{1}+\alpha_{3}-1 \\
i
\end{array}\right)\left(\begin{array}{c}
\alpha_{2}-1 \\
l
\end{array}\right) \\
& \times\left[(i+1) a+t_{1}\right]^{r} F\left(\Gamma, t_{2}\right)
\end{aligned}
$$

where: 


$$
\begin{aligned}
& F\left(\Gamma, t_{2}\right)=(-1)^{i+j+k+r+\ell+m+n} \frac{(i+1)^{j+k}(\ell+1)^{m+n} b^{j+m} c^{k+n}}{2^{j+m} 3^{k+n} j ! k ! r ! m ! n !} \\
& \times\left[\frac{a^{2} \Gamma(p+2)}{\left(p_{1}+1\right)\left[(\ell+1) a+t_{2}\right]^{p+2}}+\frac{a b\left(2 p_{1}+3\right) \Gamma(p+3)}{\left(p_{1}+1\right)\left(p_{1}+2\right)\left[(\ell+1) a+t_{2}\right]^{p+3}}\right. \\
& +\frac{a c\left(2 p_{1}+4\right) \Gamma(p+4)}{\left(p_{1}+1\right)\left(p_{1}+3\right)\left[(\ell+1) a+t_{2}\right]^{p+4}}+\frac{b^{2} \Gamma(p+4)}{\left(p_{1}+2\right)\left[(\ell+1) a+t_{2}\right]^{p+4}} \\
& \left.+\frac{b c\left(2 p_{1}+5\right) \Gamma(p+5)}{\left(p_{1}+2\right)\left(p_{1}+3\right)\left[(\ell+1) a+t_{2}\right]^{p+5}}+\frac{c^{2} \Gamma(p+6)}{\left(p_{1}+3\right)\left[(\ell+1) a+t_{2}\right]^{p+6}}\right],
\end{aligned}
$$

From Equation 10 , we can find $\mathrm{I}_{2}$, by the same way:

$$
\begin{aligned}
& I_{2}=\alpha_{1}\left(\alpha_{2}+\alpha_{3}\right) \sum_{i=0}^{\infty} \sum_{j=0}^{\infty} \sum_{k=0}^{\infty} \sum_{r=0}^{\infty} \sum_{l=0}^{\infty} \sum_{m=0}^{\infty} \sum_{n=0}^{\infty}\left(\begin{array}{c}
\alpha_{2}+\alpha_{3}-1 \\
i
\end{array}\right)\left(\begin{array}{c}
\alpha_{1}-1 \\
l
\end{array}\right) \\
& \times\left[(i+1) a+t_{2}\right]^{r} F\left(\Gamma, t_{1}\right)
\end{aligned}
$$

where:

$$
\begin{aligned}
& F\left(\Gamma, t_{1}\right)=(-1)^{i+j+k+r+\ell+m+n} \frac{(i+1)^{j+k}(\ell+1)^{m+n} b^{j+m} c^{k+n}}{2^{j+m} 3^{k+n} j ! k ! r ! m ! n !} \\
& \times\left[\frac{a^{2} \Gamma(p+2)}{\left(p_{1}+1\right)\left[(\ell+1) a+t_{1}\right]^{p+2}}+\frac{a b\left(2 p_{1}+3\right) \Gamma(p+3)}{\left(p_{1}+1\right)\left(p_{1}+2\right)\left[(\ell+1) a+t_{1}\right]^{p+3}}\right. \\
& +\frac{a c\left(2 p_{1}+4\right) \Gamma(p+4)}{\left(p_{1}+1\right)\left(p_{1}+3\right)\left[(\ell+1) a+t_{1}\right]^{p+4}}+\frac{b^{2} \Gamma(p+4)}{\left(p_{1}+2\right)\left[(\ell+1) a+t_{1}\right]^{p+4}} \\
& \left.+\frac{b c\left(2 p_{1}+5\right) \Gamma(p+5)}{\left(p_{1}+2\right)\left(p_{1}+3\right)\left[(\ell+1) a+t_{1}\right]^{p+5}}+\frac{c^{2} \Gamma(p+6)}{\left(p_{1}+3\right)\left[(\ell+1) a+t_{1}\right]^{p+6}}\right] .
\end{aligned}
$$

From Equation 11, we have:

$$
\begin{aligned}
& I_{3}=\alpha_{3} \int_{0}^{\infty} e^{-\left(t_{1}+t_{2}\right) x}\left(a+b x+c x^{2}\right) e^{-\left(a x+\frac{b}{2} x^{2}+\frac{c}{3} x^{3}\right)} \\
& \times\left[1-e^{-\left(a x+\frac{b}{2} x^{2}+\frac{c}{3} x^{3}\right)}\right]^{\alpha_{1}+\alpha_{2}+\alpha_{3}-1} d x .
\end{aligned}
$$

Since $0<e^{-\left(a x+\frac{b}{2} x^{2}+\frac{c}{3} x^{3}\right)}<1$, by using the binomial expansion for $\left[1-e^{-\left(a x+\frac{b}{2} x^{2}+\frac{c}{3} x^{3}\right)}\right]^{\alpha_{1}+\alpha_{2}+\alpha_{3}-1}$, we have:

$$
\begin{aligned}
& I_{3}=\alpha_{3} \sum_{i=0}^{\infty}\left(\begin{array}{c}
\alpha_{1}+\alpha_{2}+\alpha_{3}-1 \\
i
\end{array}\right)(-1)^{i} \int_{0}^{\infty} e^{-\left(t_{1}+t_{2}\right) x}\left(a+b x+c x^{2}\right) \\
& \times e^{-(i+1)\left(a x+\frac{b}{2} x^{2}+\frac{c}{3} x^{3}\right)} d x,
\end{aligned}
$$

By using the Taylor's expression for $e^{-(i+1) \frac{b}{2} x^{2}}$ and $e^{-(i+1) \frac{c}{3} x^{3}}$, we have:

$$
\begin{aligned}
& I_{3}=\alpha_{3} \sum_{i=0}^{\infty} \sum_{j=0}^{\infty} \sum_{k=0}^{\infty}\left(\begin{array}{c}
\alpha_{1}+\alpha_{2}+\alpha_{3}-1 \\
i
\end{array}\right)(-1)^{i+j+k} \frac{(i+1)^{j+k} b^{j} c^{k}}{2^{j} 3^{k} j ! k !} \\
& \times \int_{0}^{\infty}\left(a+b x+c x^{2}\right) x^{2 j+3 k} e^{-\left[(i+1) a+t_{1}+t_{2}\right] x} d x,
\end{aligned}
$$

Put $u=\left[(i+1) a+t_{1}+t_{2}\right] x$, then we have:

$$
\begin{aligned}
& I_{3}=\alpha_{3} \sum_{i=0}^{\infty} \sum_{j=0}^{\infty} \sum_{k=0}^{\infty}\left(\begin{array}{c}
\alpha_{1}+\alpha_{2}+\alpha_{3}-1 \\
i
\end{array}\right)(-1)^{i+j+k} \frac{(i+1)^{j+k} b^{j} c^{k}}{2^{j} 3^{k} j ! k !} \\
& \times \int_{0}^{\infty}\left[\frac{a u^{2 j+3 k}}{\left[(i+1) a+t_{1}+t_{2}\right]^{2 j+3 k+1}}+\frac{b u^{2 j+3 k+1}}{\left[(i+1) a+t_{1}+t_{2}\right]^{2 j+3 k+2}}\right. \\
& \left.+\frac{c u^{2 j+3 k+2}}{\left[(i+1) a+t_{1}+t_{2}\right]^{2 j+3 k+3}}\right] e^{-u} d u \\
& =\alpha_{3} \sum_{i=0}^{\infty} \sum_{j=0}^{\infty} \sum_{k=0}^{\infty}\left(\begin{array}{c}
\alpha_{1}+\alpha_{2}+\alpha_{3}-1 \\
i
\end{array}\right) F\left(\Gamma, t_{1}, t_{2}\right),
\end{aligned}
$$

where:

$$
\begin{aligned}
& F\left(\Gamma, t_{1}, t_{2}\right)=(-1)^{i+j+k} \frac{(i+1)^{j+k} b^{j} c^{k}}{2^{j} 3^{k} j ! k !}\left[\frac{a \Gamma(2 j+3 m+1)}{\left[(i+1) a+t_{1}+t_{2}\right]^{2 j+3 m+1}}\right. \\
& \left.+\frac{b \Gamma(2 j+3 m+2)}{\left[(i+1) a+t_{1}+t_{2}\right]^{2 j+3 m+2}}+\frac{c \Gamma(2 j+3 m+3)}{\left[(i+1) a+t_{1}+t_{2}\right]^{2 j+3 m+3}}\right] .
\end{aligned}
$$

Substituting from Equation 40-42 into Equation 37, we have Equation 36, which completes the proof.

\section{Remark 13}

The joint moment generating function $M_{X 1, X 2}\left(t_{1}, t_{2}\right)$ can be used to derive the expectation of $X_{1} X_{2}$ as:

$$
E\left[X_{1} X_{2}\right]=\left[\frac{\partial^{2}}{\partial t_{1} \partial t_{2}} M_{X_{1}, X_{2}}\left(t_{1}, t_{2}\right)\right]_{t_{1}=t_{2}=0} .
$$

\section{Maximum Likelihood Estimators}

Suppose that $\left(\left(x_{11}, x_{21}\right),\left(x_{12}, x_{22}\right), \ldots,\left(x_{1 n}, x_{2 n}\right)\right)$ is a random sample from BGQHRD with parameters $\alpha_{1}, \alpha_{2}$, $\alpha_{3}, a, b, c$. Consider that:

$$
\begin{aligned}
& n_{1}=\left(\mathrm{i} ; x_{1 \mathrm{i}}<x_{2 \mathrm{i}}\right), \quad n_{2}=\left(\mathrm{i} ; x_{1 i}>x_{2 i}\right), \\
& n_{3}=\left(\mathrm{i} ; x_{1 i}=x_{2 i}=x_{i}\right), n=n_{1}+n_{2}+n_{3} .
\end{aligned}
$$

For the sample of size $n$, the likelihood function is given by:

$$
\ell(\Phi)=\prod_{i=1}^{n_{1}} f_{1}\left(x_{1 i}, x_{2 i}\right) \prod_{i=1}^{n_{2}} f_{2}\left(x_{1 i}, x_{2 i}\right) \prod_{i=1}^{n_{3}} f_{3}\left(x_{i}, x_{i}\right)
$$

where, $\Phi=\left(\alpha_{1}, \alpha_{2}, \alpha_{3}, a, b, c\right)$.

Substituting from Equation 9-11 the likelihood function becomes: 


$$
\begin{aligned}
& \ell(\Phi)=\left(\alpha_{1}+\alpha_{3}\right)^{n_{1}}\left(\alpha_{2}+\alpha_{3}\right)^{n_{2}} \alpha_{1}^{n_{2}} \alpha_{2}^{n_{1}} \alpha_{3}^{n_{3}} \prod_{i=1}^{n_{1}}\left(a+b x_{1 i}+c x_{1 i}^{2}\right) \\
& e^{-\sum_{i=1}^{m_{1}}\left(a x_{i i}+\frac{b}{2} x_{1 i}^{2}+\frac{c}{3} x_{1 i}^{3}\right)} \prod_{i=1}^{n_{1}}\left[1-e^{-\left(a x_{1 i}+\frac{b}{2} x_{x_{i}}^{2}+\frac{c}{3} x_{1 i}^{3}\right)}\right]^{\alpha_{1}+\alpha_{3}-1} \\
& \prod_{i=1}^{n_{1}}\left(a+b x_{2 i}+c x_{2 i}^{2}\right) e^{-\sum_{i=1}^{m}\left(a x_{2 i}+\frac{b}{2} x_{2 i}^{2}+\frac{c}{3} x_{2 i}^{3}\right)} \prod_{i=1}^{n_{1}}\left[1-e^{-\left(a x_{2} i+\frac{b}{2} x_{2 i}^{2}+\frac{c}{3} x_{2 i}^{3}\right)}\right]^{\alpha_{2}-1} \\
& \prod_{i=1}^{n_{2}}\left(a+b x_{1 i}+c x_{1 i}^{2}\right) e^{-\sum_{i=1}^{n_{2}}\left(a x_{1 i}+\frac{b}{2} x_{i i}^{2}+\frac{c}{3} x_{1 i}^{3}\right)} \times \prod_{i=1}^{n_{2}}\left[1-e^{-\left(a x_{1 i}+\frac{b}{2} x_{i i}^{2}+\frac{c}{3} x_{1 i}^{3}\right)}\right]^{\alpha_{1}-1} \\
& \prod_{i=1}^{n_{2}}\left(a+b x_{2 i}+c x_{2 i}^{2}\right) e^{-\sum_{i=1}^{n_{2}}\left(a x_{2 i}+\frac{b}{2} x_{2 i}^{2}+\frac{c}{3} x_{2 i}^{3}\right)} \prod_{i=1}^{n_{2}}\left[1-e^{-\left(a x_{2 i}+\frac{b}{2} x_{2 i}^{2}+\frac{c}{3} x_{2 i}^{3}\right)}\right]^{\alpha_{2}+\alpha_{3}-1} \\
& \prod_{i=1}^{n_{3}}\left(a+b x_{i}+c x_{i}^{2}\right) e^{-\sum_{i=1}^{n_{3}}\left(a x_{i}+\frac{b}{2} x_{i}^{2}+\frac{c}{3} x_{i}^{3}\right)} \prod_{i=1}^{n_{3}}\left[1-e^{-\left(a x_{i}+\frac{b}{2} x_{i}^{2}+\frac{c}{3} x_{i}^{3}\right)}\right]^{\alpha_{1}+\alpha_{2}+\alpha_{3}-1}
\end{aligned}
$$

The log-likelihood function is given as:

$$
\begin{aligned}
& \mathcal{L}(\Phi)=n_{1} \ln \left(\alpha_{1}+\alpha_{3}\right)+n_{1} \ln \left(\alpha_{2}\right)+n_{2} \ln \left(\alpha_{2}+\alpha_{3}\right)+n_{2} \ln \left(\alpha_{1}\right) \\
& +n_{3} \ln \left(\alpha_{3}\right)+\sum_{i=1}^{n_{1}} \ln \left(a+b x_{1 i}+c x_{1 i}^{2}\right)-\sum_{i=1}^{n_{1}}\left(a x_{1 i}+\frac{b}{2} x_{1 i}^{2}+\frac{c}{3} x_{1 i}^{3}\right) \\
& +\left(\alpha_{1}+\alpha_{3}-1\right) \sum_{i=1}^{n_{1}} \ln \left[1-e^{-\left(a x_{1 i}+\frac{b}{2} x_{i i}^{2}+\frac{c}{3} x_{1 i}^{3}\right)}\right]+\sum_{i=1}^{n_{1}} \ln \left(a+b x_{2 i}+c x_{2 i}^{2}\right) \\
& -\sum_{i=1}^{n_{1}}\left(a x_{2 i}+\frac{b}{2} x_{2 i}^{2}+\frac{c}{3} x_{2 i}^{3}\right)+\left(\alpha_{2}-1\right) \sum_{i=1}^{n_{1}} \ln \left[1-e^{-\left(a x_{2 i}+\frac{b}{2} x_{2 i}^{2}+\frac{c}{3} x_{2 i}^{3}\right)}\right] \\
& +\sum_{i=1}^{n_{2}} \ln \left(a+b x_{1 i}+c x_{1 i}^{2}\right)-\sum_{i=1}^{n_{2}}\left(a x_{1 i}+\frac{b}{2} x_{1 i}^{2}+\frac{c}{3} x_{1 i}^{3}\right)+\left(\alpha_{1}-1\right) \\
& \times \sum_{i=1}^{n_{2}} \ln \left[1-e^{-\left(a x_{i i}+\frac{b}{2} x_{1 i}^{2}+\frac{c}{3} x_{i i}^{3}\right)}\right]+\sum_{i=1}^{n_{2}} \ln \left(a+b x_{2 i}+c x_{2 i}^{2}\right) \\
& -\sum_{i=1}^{n_{2}}\left(a x_{2 i}+\frac{b}{2} x_{2 i}^{2}+\frac{c}{3} x_{2 i}^{3}\right)+\left(\alpha_{2}+\alpha_{3}-1\right) \\
& \times \sum_{i=1}^{n_{2}} \ln \left[1-e^{-\left(a x_{2 i}+\frac{b}{2} x_{2 i}^{2}+\frac{c}{3} x_{2 i}^{3}\right)}\right]+\sum_{i=1}^{n_{3}} \ln \left(a+b x_{i}+c x_{i}^{2}\right) \\
& -\sum_{i=1}^{n_{3}}\left(a x_{i}+\frac{b}{2} x_{i}^{2}+\frac{c}{3} x_{i}^{3}\right)+\left(\alpha_{1}+\alpha_{2}+\alpha_{3}-1\right) \\
& \times \sum_{i=1}^{n_{3}} \ln \left[1-e^{-\left(a x_{i}+\frac{b}{2} x_{i}^{2}+\frac{c}{3} x_{i}^{3}\right)}\right] .
\end{aligned}
$$

The first partial derivatives of Equation 43 with respect to $\alpha_{1}, \alpha_{2}, \alpha_{3}, \mathrm{a}, \mathrm{b}$ and $\mathrm{c}$, can be computed as follows:

$$
\begin{aligned}
& \frac{\partial \mathcal{L}}{\partial \alpha_{1}}=\frac{n_{1}}{\alpha_{1}+\alpha_{3}}+\frac{n_{2}}{\alpha_{1}}+\sum_{i=1}^{n_{1}} \ln \left[1-e^{-\left(a x_{1 i}+\frac{b}{2} x_{1 i}^{2}+\frac{c}{3} x_{1 i}^{3}\right)}\right] \\
& +\sum_{i=1}^{n_{2}} \ln \left[1-e^{-\left(a x_{i i}+\frac{b}{2} x_{1 i}^{2}+\frac{c}{3} x_{1 i}^{3}\right)}\right]+\sum_{i=1}^{n_{3}} \ln \left[1-e^{-\left(a x_{i}+\frac{b}{2} x_{i}^{2}+\frac{c}{3} x_{i}^{3}\right)}\right],
\end{aligned}
$$

$$
\begin{aligned}
& \frac{\partial \mathcal{L}}{\partial \alpha_{2}}=\frac{n_{2}}{\alpha_{2}+\alpha_{3}}+\frac{n_{1}}{\alpha_{2}}+\sum_{i=1}^{n_{1}} \ln \left[1-e^{-\left(a x_{2 i}+\frac{b}{2} x_{2 i}^{2}+\frac{c}{3} x_{2 i}^{3}\right)}\right] \\
& +\sum_{i=1}^{n_{2}} \ln \left[1-e^{-\left(a x_{2 i}+\frac{b}{2} x_{2 i}^{2}+\frac{c}{3} x_{2 i}^{3}\right)}\right]+\sum_{i=1}^{n_{3}} \ln \left[1-e^{-\left(a x_{i}+\frac{b}{2} x_{i}^{2}+\frac{c}{3} x_{i}^{3}\right)}\right] \text {, } \\
& \frac{\partial \mathcal{L}}{\partial \alpha_{3}}=\frac{n_{1}}{\alpha_{1}+\alpha_{3}}+\frac{n_{2}}{\alpha_{2}+\alpha_{3}}+\frac{n_{3}}{\alpha_{3}}+\sum_{i=1}^{n_{1}} \ln \left[1-e^{-\left(a x_{1 i}+\frac{b}{2} x_{1 i}^{2}+\frac{c}{3} x_{1 i}^{3}\right)}\right] \\
& +\sum_{i=1}^{n_{2}} \ln \left[1-e^{-\left(a x_{2 i}+\frac{b}{2} x_{2 i}^{2}+\frac{c}{3} x_{2 i}^{3}\right)}\right]+\sum_{i=1}^{n_{3}} \ln \left[1-e^{-\left(a x_{i}+\frac{b}{2} x_{i}^{2}+\frac{c}{3} x_{i}^{3}\right)}\right], \\
& \frac{\partial \mathcal{L}}{\partial a}=\sum_{i=1}^{n_{1}} \mathcal{A}\left(x_{1 i}\right)-\sum_{i=1}^{n_{1}} x_{1 i}+\left(\alpha_{1}+\alpha_{3}-1\right) \sum_{i=1}^{n_{1}} x_{1 i} \mathcal{B}\left(x_{1 i}\right) \\
& +\sum_{i=1}^{n_{1}} \mathcal{A}\left(x_{2 i}\right)-\sum_{i=1}^{n_{1}} x_{2 i}+\left(\alpha_{2}-1\right) \sum_{i=1}^{n_{1}} x_{2 i} \mathcal{B}\left(x_{2 i}\right)+\sum_{i=1}^{n_{2}} \mathcal{A}\left(x_{1 i}\right) \\
& -\sum_{i=1}^{n_{2}} x_{1 i}+\left(\alpha_{1}-1\right) \sum_{i=1}^{n_{2}} x_{1 i} \mathcal{B}\left(x_{1 i}\right)+\sum_{i=1}^{n_{2}} \mathcal{A}\left(x_{2 i}\right)-\sum_{i=1}^{n_{2}} x_{2 i} \\
& +\left(\alpha_{2}+\alpha_{3}-1\right) \sum_{i=1}^{n_{2}} x_{2 i} \mathcal{B}\left(x_{2 i}\right)+\sum_{i=1}^{n_{3}} \mathcal{A}\left(x_{i}\right)-\sum_{i=1}^{n_{3}} x_{i} \\
& +\left(\alpha_{1}+\alpha_{2}+\alpha_{3}-1\right) \sum_{i=1}^{n_{3}} x_{i} \mathcal{B}\left(x_{i}\right) \\
& \frac{\partial \mathcal{L}}{\partial b}=\sum_{i=1}^{n_{1}} x_{1 i} \mathcal{A}\left(x_{1 i}\right)-\frac{1}{2} \sum_{i=1}^{n_{1}} x_{1 i}^{2}+\frac{1}{2}\left(\alpha_{1}+\alpha_{3}-1\right) \sum_{i=1}^{n_{1}} x_{1 i}^{2} \mathcal{B}\left(x_{1 i}\right) \\
& +\sum_{i=1}^{n_{1}} x_{2 i} \mathcal{A}\left(x_{2 i}\right)-\frac{1}{2} \sum_{i=1}^{n_{1}} x_{2 i}^{2}+\frac{1}{2}\left(\alpha_{2}-1\right) \sum_{i=1}^{n_{1}} x_{2 i}^{2} \mathcal{B}\left(x_{2 i}\right) \\
& +\sum_{i=1}^{n_{2}} x_{1 i} \mathcal{A}\left(x_{1 i}\right)-\frac{1}{2} \sum_{i=1}^{n_{2}} x_{1 i}^{2}+\frac{1}{2}\left(\alpha_{1}-1\right) \sum_{i=1}^{n_{2}} x_{1 i}^{2} \mathcal{B}\left(x_{1 i}\right) \\
& +\sum_{i=1}^{n_{2}} x_{2 i} \mathcal{A}\left(x_{2 i}\right)-\frac{1}{2} \sum_{i=1}^{n_{2}} x_{2 i}^{2}+\frac{1}{2}\left(\alpha_{2}+\alpha_{3}-1\right) \sum_{i=1}^{n_{2}} x_{2 i}^{2} \mathcal{B}\left(x_{2 i}\right) \\
& +\sum_{i=1}^{n_{3}} x_{i} \mathcal{A}\left(x_{i}\right)-\frac{1}{2} \sum_{i=1}^{n_{3}} x_{i}^{2}+\frac{1}{2}\left(\alpha_{1}+\alpha_{2}+\alpha_{3}-1\right) \sum_{i=1}^{n_{3}} x_{i}^{2} \mathcal{A}\left(x_{i}\right), \\
& \frac{\partial \mathcal{L}}{\partial c}=\sum_{i=1}^{n_{1}} x_{1 i}^{2} \mathcal{A}\left(x_{1 i}\right)-\frac{1}{3} \sum_{i=1}^{n_{1}} x_{1 i}^{3}+\frac{1}{3}\left(\alpha_{1}+\alpha_{3}-1\right) \sum_{i=1}^{n_{1}} x_{1 i}^{3} \mathcal{B}\left(x_{1 i}\right) \\
& +\sum_{i=1}^{n_{1}} x_{2 i}^{2} \mathcal{A}\left(x_{2 i}\right)-\frac{1}{3} \sum_{i=1}^{n_{1}} x_{2 i}^{3}+\frac{1}{3}\left(\alpha_{2}-1\right) \sum_{i=1}^{n_{1}} x_{2 i}^{3} \mathcal{B}\left(x_{2 i}\right) \\
& +\sum_{i=1}^{n_{2}} x_{1 i}^{2} \mathcal{A}\left(x_{1 i}\right)-\frac{1}{3} \sum_{i=1}^{n_{2}} x_{1 i}^{3}+\frac{1}{3}\left(\alpha_{1}-1\right) \sum_{i=1}^{n_{2}} x_{1 i}^{3} \mathcal{B}\left(x_{1 i}\right) \\
& +\sum_{i=1}^{n_{2}} x_{2 i}^{2} \mathcal{A}\left(x_{2 i}\right)-\frac{1}{3} \sum_{i=1}^{n_{2}} x_{2 i}^{3}+\frac{1}{3}\left(\alpha_{2}+\alpha_{3}-1\right) \sum_{i=1}^{n_{2}} x_{2 i}^{3} \mathcal{B}\left(x_{2 i}\right) \\
& +\sum_{i=1}^{n_{3}} x_{i}^{2} \mathcal{A}\left(x_{i}\right)-\frac{1}{3} \sum_{i=1}^{n_{3}} x_{i}^{3}+\frac{1}{3}\left(\alpha_{1}+\alpha_{2}+\alpha_{3}-1\right) \sum_{i=1}^{n_{3}} x_{i}^{3} \mathcal{B}\left(x_{i}\right),
\end{aligned}
$$

where:

$$
\mathcal{A}(x)=\frac{1}{a+b x+c x^{2}}, \quad \mathcal{B}(x)=\frac{e^{-\left(a x+\frac{b}{2} x^{2}+\frac{c}{3} x^{3}\right)}}{1-e^{-\left(a x+\frac{b}{2} x^{2}+\frac{c}{3} x^{3}\right)}} .
$$


To get the MLEs of the parameters $\alpha_{1}, \alpha_{2}, \alpha_{3}, a, b$ and $c$, we have to solve the above system on nonlinear equation with respect to $\alpha_{1}, \alpha_{2}, \alpha_{3}, a, b$ and $c$. The solution of the Equation 44 to 49 are not easy to solve. Therefore a numerical technique can be used to get the MLEs.

Based on the asymptotic distributions of the MLEs the approximate confidence intervals of the parameters are derived. We determine the second partial derivatives to obtain the information matrix for $\alpha_{1}, \alpha_{2}, \alpha_{3}, a, b$ and $c$ as follows:

$\frac{\partial^{2} \mathcal{L}}{\partial \alpha_{1}^{2}}=-\frac{n_{1}}{\left(\alpha_{1}+\alpha_{3}\right)^{2}}-\frac{n_{2}}{\alpha_{1}^{2}}$

$\frac{\partial^{2} \mathcal{L}}{\partial \alpha_{1} \partial \alpha_{2}}=0$

$\frac{\partial^{2} \mathcal{L}}{\partial \alpha_{1} \partial \alpha_{3}}=-\frac{n_{1}}{\left(\alpha_{1}+\alpha_{3}\right)^{2}}$,

$\frac{\partial^{2} \mathcal{L}}{\partial \alpha_{1} \partial a}=\sum_{i=1}^{n_{1}} x_{1 i} \mathcal{B}\left(x_{1 i}\right)+\sum_{i=1}^{n_{2}} x_{1 i} \mathcal{B}\left(x_{1 i}\right)+\sum_{i=1}^{n_{3}} x_{i} \mathcal{B}\left(x_{i}\right)$

$\frac{\partial^{2} \mathcal{L}}{\partial \alpha_{1} \partial b}=\frac{1}{2}\left[\sum_{i=1}^{n_{1}} x_{1 i}^{2} \mathcal{B}\left(x_{1 i}\right)+\sum_{i=1}^{n_{2}} x_{1 i}^{2} \mathcal{B}\left(x_{1 i}\right)+\sum_{i=1}^{n_{3}} x_{i}^{2} \mathcal{B}\left(x_{i}\right)\right]$,

$\frac{\partial^{2} \mathcal{L}}{\partial \alpha_{1} \partial c}=\frac{1}{3}\left[\sum_{i=1}^{n_{1}} x_{1 i}^{3} \mathcal{B}\left(x_{1 i}\right)+\sum_{i=1}^{n_{2}} x_{1 i}^{3} \mathcal{B}\left(x_{1 i}\right)+\sum_{i=1}^{n_{3}} x_{i}^{3} \mathcal{B}\left(x_{i}\right)\right]$,

$\frac{\partial^{2} \mathcal{L}}{\partial \alpha_{2}^{2}}=-\frac{n_{2}}{\left(\alpha_{2}+\alpha_{3}\right)^{2}}-\frac{n_{1}}{\alpha_{2}^{2}}$

$\frac{\partial^{2} \mathcal{L}}{\partial \alpha_{2} \partial \alpha_{3}}=-\frac{n_{2}}{\left(\alpha_{2}+\alpha_{3}\right)^{2}}$,

$\frac{\partial^{2} \mathcal{L}}{\partial \alpha_{2} \partial a}=\sum_{i=1}^{n_{1}} x_{2 i} \mathcal{B}\left(x_{2 i}\right)+\sum_{i=1}^{n_{2}} x_{2 i} \mathcal{B}\left(x_{2 i}\right)+\sum_{i=1}^{n_{3}} x_{i} \mathcal{B}\left(x_{i}\right)$,

$\frac{\partial^{2} \mathcal{L}}{\partial \alpha_{2} \partial b}=\frac{1}{2}\left[\sum_{i=1}^{n_{1}} x_{2 i}^{2} \mathcal{B}\left(x_{2 i}\right)+\sum_{i=1}^{n_{2}} x_{2 i}^{2} \mathcal{B}\left(x_{2 i}\right)+\sum_{i=1}^{n_{3}} x_{i}^{2} \mathcal{B}\left(x_{i}\right)\right]$,

$\frac{\partial^{2} \mathcal{L}}{\partial \alpha_{2} \partial c}=\frac{1}{3}\left[\sum_{i=1}^{n_{1}} x_{2 i}^{3} \mathcal{B}\left(x_{2 i}\right)+\sum_{i=1}^{n_{2}} x_{2 i}^{3} \mathcal{B}\left(x_{2 i}\right)+\sum_{i=1}^{n_{3}} x_{i}^{3} \mathcal{B}\left(x_{i}\right)\right]$,

$\frac{\partial^{2} \mathcal{L}}{\partial \alpha_{3}^{2}}=-\frac{n_{1}}{\left(\alpha_{1}+\alpha_{3}\right)^{2}}-\frac{n_{2}}{\left(\alpha_{2}+\alpha_{3}\right)^{2}}-\frac{n_{3}}{\alpha_{3}^{2}}$

$\frac{\partial^{2} \mathcal{L}}{\partial \alpha_{3} \partial a}=\sum_{i=1}^{n_{1}} x_{1 i} \mathcal{B}\left(x_{1 i}\right)+\sum_{i=1}^{n_{2}} x_{2 i} \mathcal{B}\left(x_{2 i}\right)+\sum_{i=1}^{n_{3}} x_{i} \mathcal{B}\left(x_{i}\right)$, $\frac{\partial^{2} \mathcal{L}}{\partial \alpha_{3} \partial b}=\frac{1}{2}\left[\sum_{i=1}^{n_{1}} x_{1 i}^{2} \mathcal{B}\left(x_{1 i}\right)+\sum_{i=1}^{n_{2}} x_{2 i}^{2} \mathcal{B}\left(x_{2 i}\right)+\sum_{i=1}^{n_{3}} x_{i}^{2} \mathcal{B}\left(x_{i}\right)\right]$,

$\frac{\partial^{2} \mathcal{L}}{\partial \alpha_{3} \partial c}=\frac{1}{3}\left[\sum_{i=1}^{n_{1}} x_{1 i}^{3} \mathcal{B}\left(x_{1 i}\right)+\sum_{i=1}^{n_{2}} x_{2 i}^{3} \mathcal{B}\left(x_{2 i}\right)+\sum_{i=1}^{n_{3}} x_{i}^{3} \mathcal{B}\left(x_{i}\right)\right]$,

$\frac{\partial \mathcal{L}}{\partial a^{2}}=-\sum_{i=1}^{n_{1}} \mathcal{A}^{2}\left(x_{1 i}\right)-\left(\alpha_{1}+\alpha_{3}-1\right) \sum_{i=1}^{n_{1}} x_{1 i}^{2} \mathcal{C}\left(x_{1 i}\right)-\sum_{i=1}^{n_{1}} \mathcal{A}^{2}\left(x_{2 i}\right)$

$-\left(\alpha_{2}-1\right) \sum_{i=1}^{n_{1}} x_{2 i}^{2} \mathcal{C}\left(x_{2 i}\right)-\sum_{i=1}^{n_{2}} \mathcal{A}^{2}\left(x_{1 i}\right)-\left(\alpha_{1}-1\right) \sum_{i=1}^{n_{2}} x_{1 i}^{2} \mathcal{C}\left(x_{1 i}\right)$

$-\sum_{i=1}^{n_{2}} \mathcal{A}^{2}\left(x_{2 i}\right)-\left(\alpha_{2}+\alpha_{3}-1\right) \sum_{i=1}^{n_{2}} x_{2 i}^{2} \mathcal{C}\left(x_{2 i}\right)-\sum_{i=1}^{n_{3}} \mathcal{A}^{2}\left(x_{i}\right)$

$-\left(\alpha_{1}+\alpha_{2}+\alpha_{3}-1\right) \sum_{i=1}^{n_{3}} x_{i}^{2} \mathcal{C}\left(x_{i}\right)$,

$\frac{\partial \mathcal{L}}{\partial a \partial b}=-\sum_{i=1}^{n_{1}} x_{i 1} \mathcal{A}^{2}\left(x_{1 i}\right)-\frac{1}{2}\left(\alpha_{1}+\alpha_{3}-1\right) \sum_{i=1}^{n_{1}} x_{1 i}^{3} \mathcal{C}\left(x_{1 i}\right)$

$-\sum_{i=1}^{n_{1}} x_{2 i} \mathcal{A}^{2}\left(x_{2 i}\right)-\frac{1}{2}\left(\alpha_{2}-1\right) \sum_{i=1}^{n_{1}} x_{2 i}^{3} \mathcal{C}\left(x_{2 i}\right)-\sum_{i=1}^{n_{2}} x_{1 i} \mathcal{A}^{2}\left(x_{1 i}\right)$

$-\frac{1}{2}\left(\alpha_{1}-1\right) \sum_{i=1}^{n_{2}} x_{1 i}^{3} \mathcal{C}\left(x_{1 i}\right)-\sum_{i=1}^{n_{2}} x_{2 i} \mathcal{A}^{2}\left(x_{2 i}\right)-\frac{1}{2}\left(\alpha_{2}+\alpha_{3}-1\right)$

$\times \sum_{i=1}^{n_{2}} x_{2 i}^{3} \mathcal{C}\left(x_{2 i}\right)-\sum_{i=1}^{n_{3}} x_{i} \mathcal{A}^{2}\left(x_{i}\right)-\frac{1}{2}\left(\alpha_{1}+\alpha_{2}+\alpha_{3}-1\right) \sum_{i=1}^{n_{3}} x_{i}^{3} \mathcal{C}\left(x_{i}\right)$,

$\frac{\partial \mathcal{L}}{\partial a \partial c}=-\sum_{i=1}^{n_{1}} x_{i 1}^{2} \mathcal{A}^{2}\left(x_{1 i}\right)-\frac{1}{3}\left(\alpha_{1}+\alpha_{3}-1\right) \sum_{i=1}^{n_{1}} x_{1 i}^{4} \mathcal{C}\left(x_{1 i}\right)$

$-\sum_{i=1}^{n_{1}} x_{2 i}^{2} \mathcal{A}^{2}\left(x_{2 i}\right)-\frac{1}{3}\left(\alpha_{2}-1\right) \sum_{i=1}^{n_{1}} x_{2 i}^{4} \mathcal{C}\left(x_{2 i}\right)-\sum_{i=1}^{n_{2}} x_{1 i}^{2} \mathcal{A}^{2}\left(x_{1 i}\right)$

$-\frac{1}{3}\left(\alpha_{1}-1\right) \sum_{i=1}^{n_{2}} x_{1 i}^{4} \mathcal{C}\left(x_{1 i}\right)-\sum_{i=1}^{n_{2}} x_{2 i}^{2} \mathcal{A}^{2}\left(x_{2 i}\right)-\frac{1}{3}\left(\alpha_{2}+\alpha_{3}-1\right)$

$\times \sum_{i=1}^{n_{2}} x_{2 i}^{4} \mathcal{C}\left(x_{2 i}\right)-\sum_{i=1}^{n_{3}} x_{i}^{2} \mathcal{A}^{2}\left(x_{i}\right)-\frac{1}{3}\left(\alpha_{1}+\alpha_{2}+\alpha_{3}-1\right) \sum_{i=1}^{n_{3}} x_{i}^{4} \mathcal{C}\left(x_{i}\right)$,

$\frac{\partial \mathcal{L}}{\partial b^{2}}=-\sum_{i=1}^{n_{1}} x_{i 1}^{2} \mathcal{A}^{2}\left(x_{1 i}\right)-\frac{1}{4}\left(\alpha_{1}+\alpha_{3}-1\right) \sum_{i=1}^{n_{1}} x_{1 i}^{4} \mathcal{C}\left(x_{1 i}\right)$

$-\sum_{i=1}^{n_{1}} x_{2 i}^{2} \mathcal{A}^{2}\left(x_{2 i}\right)-\frac{1}{4}\left(\alpha_{2}-1\right) \sum_{i=1}^{n_{1}} x_{2 i}^{4} \mathcal{C}\left(x_{2 i}\right)-\sum_{i=1}^{n_{2}} x_{1 i}^{2} \mathcal{A}^{2}\left(x_{1 i}\right)$

$-\frac{1}{4}\left(\alpha_{1}-1\right) \sum_{i=1}^{n_{2}} x_{1 i}^{4} \mathcal{C}\left(x_{1 i}\right)-\sum_{i=1}^{n_{2}} x_{2 i}^{2} \mathcal{A}^{2}\left(x_{2 i}\right)-\frac{1}{4}\left(\alpha_{2}+\alpha_{3}-1\right)$

$\times \sum_{i=1}^{n_{2}} x_{2 i}^{4} \mathcal{C}\left(x_{2 i}\right)-\sum_{i=1}^{n_{3}} x_{i}^{2} \mathcal{A}^{2}\left(x_{i}\right)-\frac{1}{4}\left(\alpha_{1}+\alpha_{2}+\alpha_{3}-1\right) \sum_{i=1}^{n_{3}} x_{i}^{4} \mathcal{C}\left(x_{i}\right)$,

$\frac{\partial \mathcal{L}}{\partial b \partial c}=-\sum_{i=1}^{n_{1}} x_{1 i}^{3} \mathcal{A}^{2}\left(x_{1 i}\right)-\frac{1}{6}\left(\alpha_{1}+\alpha_{3}-1\right) \sum_{i=1}^{n_{1}} x_{1 i}^{5} \mathcal{C}\left(x_{1 i}\right)$

$-\sum_{i=1}^{m_{1}} x_{2 i}^{3} \mathcal{A}^{2}\left(x_{2 i}\right)-\frac{1}{6}\left(\alpha_{2}-1\right) \sum_{i=1}^{m_{1}} x_{2 i}^{5} \mathcal{C}\left(x_{2 i}\right)-\sum_{i=1}^{n_{2}} x_{1 i}^{3} \mathcal{A}^{2}\left(x_{1 i}\right)$

$-\frac{1}{6}\left(\alpha_{1}-1\right) \sum_{i=1}^{n_{2}} x_{1 i}^{5} \mathcal{C}\left(x_{1 i}\right)-\sum_{i=1}^{n_{2}} x_{2 i}^{3} \mathcal{A}^{2}\left(x_{2 i}\right)-\frac{1}{6}\left(\alpha_{2}+\alpha_{3}-1\right)$

$\times \sum_{i=1}^{n_{2}} x_{2 i}^{5} \mathcal{C}\left(x_{2 i}\right)-\sum_{i=1}^{n_{3}} x_{i}^{3} x_{i} \mathcal{A}^{2}\left(x_{i}\right)-\frac{1}{6}\left(\alpha_{1}+\alpha_{2}+\alpha_{3}-1\right) \sum_{i=1}^{n_{3}} x_{i}^{5} \mathcal{C}\left(x_{i}\right)$, 
$\frac{\partial \mathcal{L}}{\partial c^{2}}=-\sum_{i=1}^{n_{1}} x_{i 1}^{4} \mathcal{A}^{2}\left(x_{1 i}\right)-\frac{1}{9}\left(\alpha_{1}+\alpha_{3}-1\right) \sum_{i=1}^{n_{1}} x_{1 i}^{6} \mathcal{C}\left(x_{1 i}\right)$

$-\sum_{i=1}^{n_{1}} x_{2 i}^{4} \mathcal{A}^{2}\left(x_{2 i}\right)-\frac{1}{9}\left(\alpha_{2}-1\right) \sum_{i=1}^{n_{1}} x_{2 i}^{6} \mathcal{C}\left(x_{2 i}\right)-\sum_{i=1}^{n_{2}} x_{1 i}^{4} \mathcal{A}^{2}\left(x_{1 i}\right)$

$-\frac{1}{9}\left(\alpha_{1}-1\right) \sum_{i=1}^{n_{2}} x_{1 i}^{6} \mathcal{C}\left(x_{1 i}\right)-\sum_{i=1}^{n_{2}} x_{2 i}^{4} \mathcal{A}^{2}\left(x_{2 i}\right)-\frac{1}{9}\left(\alpha_{2}+\alpha_{3}-1\right)$

$\times \sum_{i=1}^{n_{2}} x_{2 i}^{6} \mathcal{C}\left(x_{2 i}\right)-\sum_{i=1}^{n_{3}} x_{i}^{4} \mathcal{A}^{2}\left(x_{i}\right)-\frac{1}{9}\left(\alpha_{1}+\alpha_{2}+\alpha_{3}-1\right) \sum_{i=1}^{n_{3}} x_{i}^{6} \mathcal{C}\left(x_{i}\right)$,

where:

$$
\mathcal{C}(x)=\frac{e^{-\left(a x+\frac{b}{2} x^{2}+\frac{c}{3} x^{3}\right)}}{\left[1-e^{-\left(a x+\frac{b}{2} x^{2}+\frac{c}{3} x^{3}\right)}\right]^{2}}
$$

Then the variance-covariance matrix may be approximated by:

$$
V=I^{-1}=\left(\begin{array}{llllll}
I_{11} & I_{12} & I_{13} & I_{14} & I_{15} & I_{16} \\
I_{21} & I_{22} & I_{23} & I_{24} & I_{25} & I_{26} \\
I_{31} & I_{32} & I_{33} & I_{34} & I_{35} & I_{36} \\
I_{41} & I_{42} & I_{43} & I_{44} & I_{45} & I_{46} \\
I_{51} & I_{52} & I_{53} & I_{54} & I_{55} & I_{56} \\
I_{61} & I_{62} & I_{63} & I_{64} & I_{65} & I_{66}
\end{array}\right)^{-1},
$$

where, $I$ is the observed information matrix and:

$$
I_{i j}=E\left[-\frac{\partial^{2} L}{\partial \theta \partial \theta^{\prime}}\right], \quad \text { where } \theta, \theta^{`}=\alpha_{1}, \alpha_{2}, \alpha_{3}, a, b, c .
$$

Using Equation 71, a $(1-\gamma) 100 \%$ confidence interval for $\alpha_{1}, \alpha_{2}, \alpha_{3}, \mathrm{a}, \mathrm{b}$ and $\mathrm{c}$ are approximated respectively as:

$$
\begin{array}{cl}
\alpha_{i} \pm z_{\frac{\gamma}{2}} \sqrt{V_{\alpha_{i} \alpha_{i}}}, & i=1,2,3, \quad a \pm z_{\frac{\gamma}{2}} \sqrt{V_{a a}} \\
b \pm z_{\frac{\gamma}{2}} \sqrt{V_{b b}}, & c \pm z_{\frac{\gamma}{2}} \sqrt{V_{c c}}
\end{array}
$$

where, $z_{\gamma / 2}$ is the upper $(\gamma / 2) t h$ percentile of the standard normal distribution.

\section{Data Analysis}

The following data represent the American Football (National Football League) League data and they are obtained from the matches played on three consecutive weekends in 1986.

The data (scoring times in minutes and seconds) are represented in the following Table 1 .

From this data, we find that the values of $\hat{\alpha}_{i}, i=1,2,3, \hat{a}, \hat{b}$ and $\hat{c}$ are $0.05,0.75,1.547,0.151$, $-5.842 \times 10^{-3}$ and $1.065 \times 10^{-4}$, respectively and the loglikelihood equals (-239.318).

By substituting the MLEs of these parameters in Equation 71, we obtain the estimation of the variance covariance matrix as:

$$
\left(\begin{array}{cccccc}
1.272 \times 10^{-3} & 1.0362 \times 10^{-3} & 1.6923 \times 10^{-3} & 2.7367 \times 10^{-4} & -2.4462 \times 10^{-5} & 4.3822 \times 10^{-7} \\
1.0362 \times 10^{-3} & 0.0725 & 0.0784 & 9.9959 \times 10^{-3} & -8.8618 \times 10^{-4} & 1.5847 \times 10^{-5} \\
1.6923 \times 10^{-3} & 0.0784 & 0.2262 & 0.021 & -1.883 \times 10^{-3} & 3.3742 \times 10^{-5} \\
2.7367 \times 10^{-4} & 9.9959 \times 10^{-3} & 0.021 & 2.722 \times 10^{-3} & -2.5713 \times 10^{-4} & 4.723 \times 10^{-6} \\
-2.4462 \times 10^{-5} & -8.8618 \times 10^{-4} & -1.883 \times 10^{-3} & -2.5713 \times 10^{-4} & 2.7573 \times 10^{-5} & -5.37 \times 10^{-7} \\
4.3822 \times 10^{-7} & 1.5847 \times 10^{-5} & 3.3742 \times 10^{-5} & 4.723 \times 10^{-6} & -5.37 \times 10^{-7} & 1.114 \times 10^{-8}
\end{array}\right) .
$$

Table 1. American Football (National Football League) League data

\begin{tabular}{lrrrrr}
\hline$X_{1}$ & $X_{2}$ & $X_{1}$ & $X_{2}$ & $X_{1}$ & $X_{2}$ \\
\hline 2.05 & 3.98 & 5.78 & 25.98 & 10.40 & 10.25 \\
9.05 & 9.05 & 13.8 & 49.75 & 2.98 & 2.98 \\
0.85 & 0.85 & 7.25 & 7.25 & 3.88 & 6.43 \\
3.43 & 3.43 & 4.25 & 4.25 & 0.75 & 0.75 \\
7.78 & 7.78 & 1.65 & 1.65 & 11.63 & 1.37 \\
10.57 & 14.28 & 6.42 & 15.08 & 10.53 & 1.38 \\
7.05 & 7.05 & 4.22 & 9.48 & 12.13 & 10.53 \\
2.58 & 2.58 & 15.53 & 15.53 & 14.58 & 12.13 \\
7.23 & 9.68 & 2.90 & 2.90 & 11.82 & 14.58 \\
6.85 & 34.58 & 7.02 & 7.02 & 5.52 & 11.82 \\
32.45 & 42.35 & 6.42 & 6.42 & 19.65 & 11.27 \\
8.53 & 14.57 & 8.98 & 8.98 & 17.83 & 10.70 \\
31.13 & 49.88 & 10.15 & 10.15 & 10.85 & 17.83 \\
14.58 & 20.57 & 8.87 & 8.87 & 38.07 \\
\hline
\end{tabular}




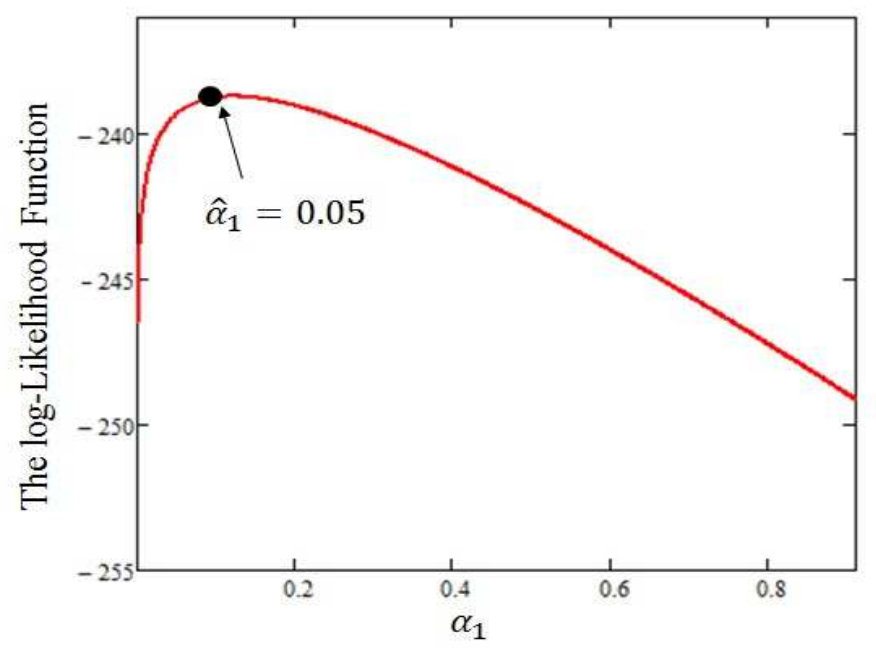

Fig. 1. The profile of the log-likelihood function of $\alpha_{1}$

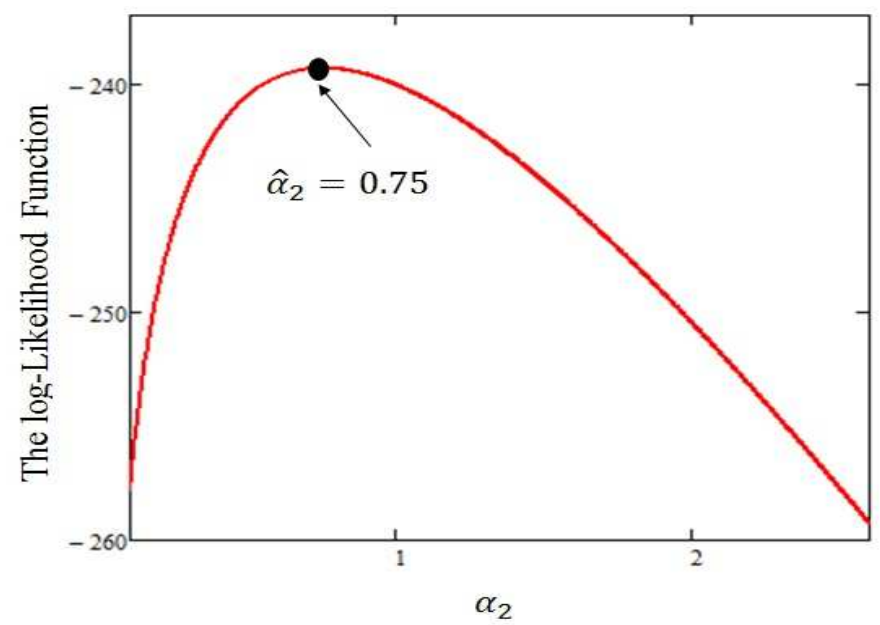

Fig. 2. The profile of the log-likelihood function of $\alpha_{2}$

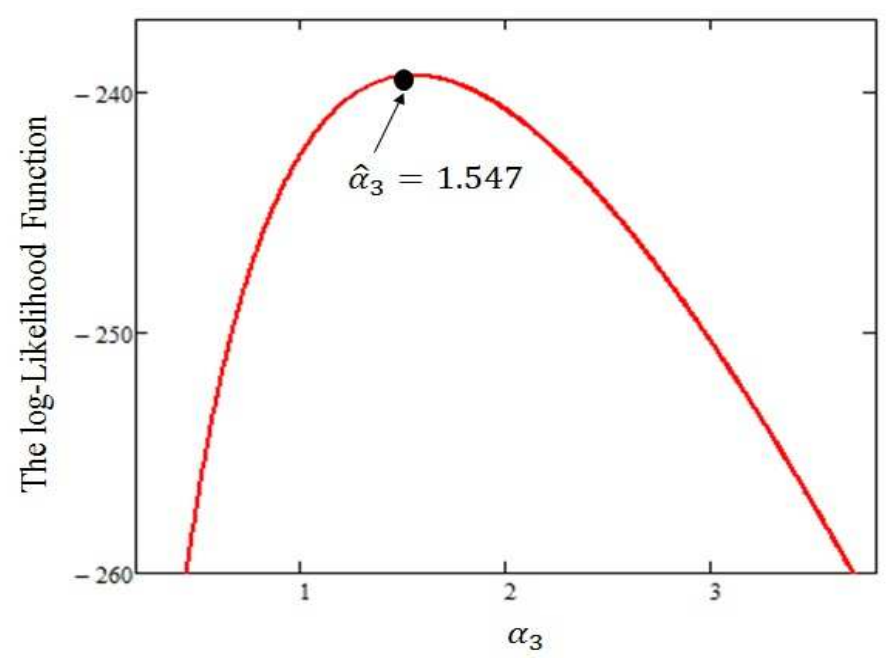

Fig. 3. The profile of the log-likelihood function of $\alpha_{3}$ 


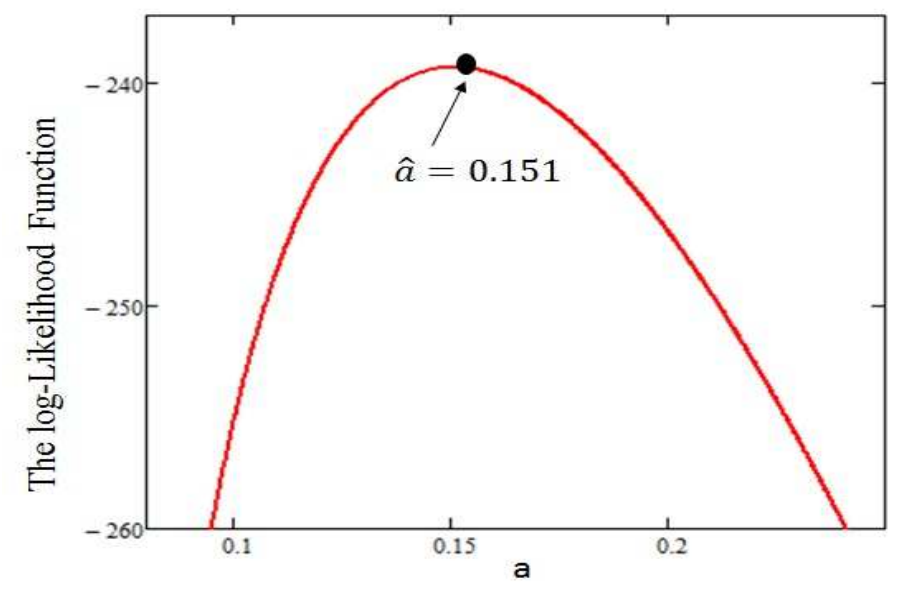

Fig. 4. The profile of the log-likelihood function of a

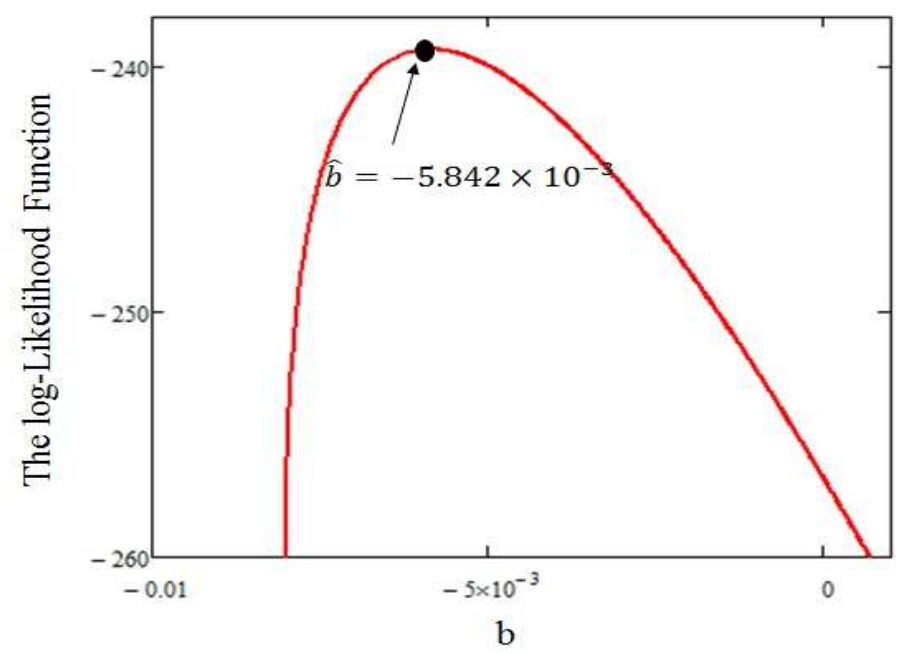

Fig. 5. The profile of the log-likelihood function of $b$

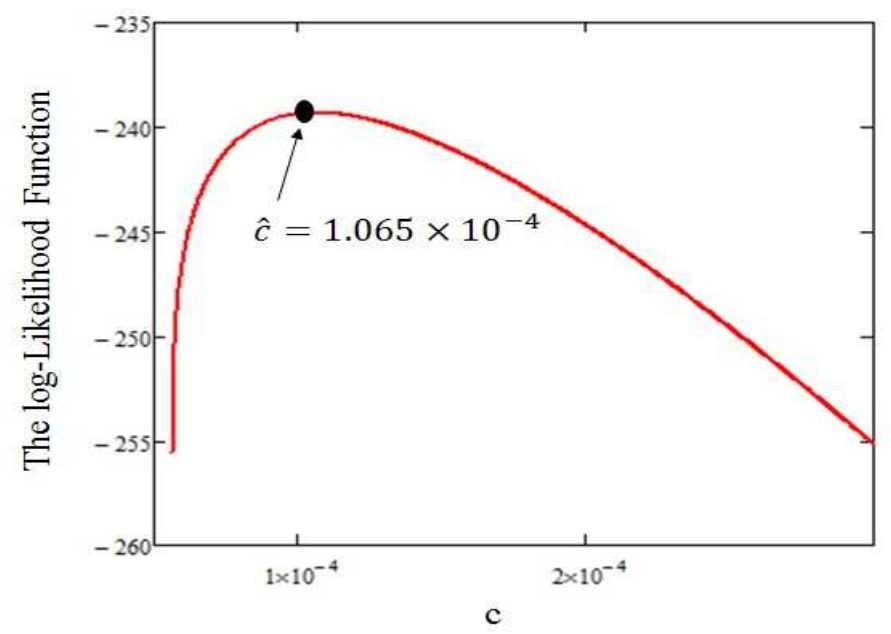

Fig. 6. The profile of the log-likelihood function of $\mathrm{c}$ 
The approximated $95 \%$ confidence interval of $\alpha_{1}, \alpha_{2}$, $\alpha_{3}, \mathrm{a}, \mathrm{b}$ and $\mathrm{c}$ are given by [0, 0.1199], [0.22237, $1.27763],[0.61491,2.47909]$, [0.04874, 0.25326], [$\left.0.01613,4.45001 \times 10^{-3}\right]$ and $\left[0,3.13368 \times 10^{-4}\right]$, respectively.

We plot the profiles of the log-likelihood function of $\alpha_{1}, \alpha_{2}, \alpha_{3}, a, b$ and c, respectively, Fig. 1-6.

\section{Conclusion}

In this study, we investigate the BGQHRD. Also, we determine the joint probability density function, the joint cumulative distribution function and joint survival distribution function. Moreover, several properties of this distribution have been discussed. Conditional probability density functions, $r$ th moments and the joint and marginal moment generating functions are derived. Parameters estimators using the maximum likelihood method are discussed. A numerical illustration by using real data is used to obtain Maximum Likelihood Estimators (MLEs) and the behavior of the estimators numerically is studied.

\section{Ethics}

The author declares that there is no conflict of interests regarding the publication of this article.

\section{References}

Al-Khedhairi, A. and A., El-Gohary, 2008. A new class of bivariate Gompertz distributions and its mixture. Int. J. Math. Anal., 2: 235-253.

Bain, L.J., 1974. Analysis for the linear failure-rate lifetesting distribution. Technometrics, 16: 551-559. DOI: 10.1080/00401706.1974.10489237

El-Sherpieny, E.A., S.A. Ibrahim and R.E. Bedar, 2013. A new bivariate distribution with generalized gompertz marginals. Asian J. Applied Sci., 1: 2321-0893.

Kundu, D. and R.D. Gupta, 2009. Bivariate generalized exponential distribution. J. Multivariate Anal., 100: 581-593. DOI: 10.1016/j.jmva.2008.06.012

Kundu, D. and K. Gupta, 2013. Bayes estimation for the Marshall-Olkin bivariate Weibull distribution. J. Comput. Stat. Data Anal., 57: 271-281. DOI: 10.1016/j.csda.2012.06.002

Marshall, A.W. and I.A. Olkin, 1986. A multivariate exponential distribution. J. Am. Stat. Assoc., 62: 30-44. DOI: 10.1080/01621459.1967.10482885

Sarhan, A., 2009. Generalized quadratic hazard rate distribution. Int. J. Applied Math. Stat., 14: 94-109.

Sarhan, A. and N. Balakrishnan, 2007. A new class of bivariate distributions and its mixture. J. Multivariate Anal., 98: 1508-1527.

DOI: $10.1016 /$ j.jmva.2006.07.007 